\title{
Search for $W$ Boson Decays to Three Charged Pions
}

\author{
A. M. Sirunyan et al. ${ }^{*}$ \\ (CMS Collaboration)
}

(Received 30 January 2019; published 18 April 2019)

\begin{abstract}
For the first time, a search for the rare decay of the $W$ boson to three charged pions has been performed. Proton-proton collision data recorded by the CMS experiment at a center-of-mass energy of $13 \mathrm{TeV}$, corresponding to an integrated luminosity of $77.3 \mathrm{fb}^{-1}$, have been analyzed. No significant excess is observed above the background expectation. An upper limit of $1.01 \times 10^{-6}$ is set at $95 \%$ confidence level on the branching fraction of the $W$ boson to three charged pions. This provides a strong motivation for theoretical calculations of this branching fraction.
\end{abstract}

DOI: 10.1103/PhysRevLett.122.151802

Exclusive decays of the $W$ boson to hadrons, if observed, would provide a new precision measurement of the mass of the $W$ boson that is based solely on visible decay products. This as yet unobserved probe of the strong interaction at the boundary of perturbative and nonperturbative domains could also provide insight into quantum chromodynamics (QCD), in particular factorization and meson form factors at high energy scales [1-3]. Upper limits on the branching fractions $(\mathcal{B})$ of two exclusive $W$ boson decays were set previously at a $95 \%$ confidence level (CL): $\mathcal{B}\left(W^{ \pm} \rightarrow\right.$ $\left.D_{s}^{ \pm} \gamma\right)<1.3 \times 10^{-3}[4]$ and $\mathcal{B}\left(W^{ \pm} \rightarrow \pi^{ \pm} \gamma\right)<7.0 \times 10^{-6}$ [5]. For the $Z$ boson, there are many more results on searches for such rare exclusive decays [6]. The decay $W^{ \pm} \rightarrow \pi^{ \pm} \pi^{ \pm} \pi^{\mp}$ could be the first exclusive decay of the $W$ boson to hadrons to be observed because the low particle multiplicity in the final state provides a clean signature in a collider experiment. There exists no theoretical calculation of $\mathcal{B}\left(W^{ \pm} \rightarrow \pi^{ \pm} \pi^{ \pm} \pi^{\mp}\right)$, but it is expected to be the same order of magnitude as $\mathcal{B}\left(Z \rightarrow \pi^{+} \pi^{-} \pi^{0}\right)$, and within the range $10^{-8}$ and $10^{-5}[2,7]$.

This Letter reports a first search for the rare $W^{ \pm} \rightarrow$ $\pi^{ \pm} \pi^{ \pm} \pi^{\mp}$ decay. The analysis relies on an innovative use of algorithms designed to trigger on and identify $\tau \rightarrow$ hadrons $+\nu_{\tau}$ weak decays $[8,9]$. The data correspond to an integrated luminosity of $77.3 \mathrm{fb}^{-1}$ recorded in protonproton $(p p)$ collisions at a center-of-mass energy of $13 \mathrm{TeV}$ in $2016\left(35.9 \mathrm{fb}^{-1}\right)$ and $2017\left(41.4 \mathrm{fb}^{-1}\right)$. The two datataking periods are analyzed separately and then combined. For simplicity, in the following the charges of the particles are omitted.

*Full author list given at the end of the Letter.

Published by the American Physical Society under the terms of the Creative Commons Attribution 4.0 International license. Further distribution of this work must maintain attribution to the author(s) and the published article's title, journal citation, and DOI. Funded by SCOAP ${ }^{3}$.
The central feature of the CMS apparatus is a superconducting solenoid $6 \mathrm{~m}$ in internal diameter, providing a magnetic field of $3.8 \mathrm{~T}$. A silicon pixel and strip tracker, a lead tungstate crystal electromagnetic calorimeter (ECAL), and a brass and scintillator hadron calorimeter reside within the solenoid volume. Each of these systems is composed of a barrel and two end cap sections. Forward hadron calorimeters extend the pseudorapidity $(\eta)$ coverage provided by the barrel and end cap detectors. Muons are detected in gas-ionization chambers embedded in the steel flux-return yoke outside the solenoid. Events of interest are selected using a two-tiered trigger system [10]. A more detailed description of the CMS detector, together with a definition of its coordinate system and relevant kinematic variables, can be found in Ref. [11].

The signal process $W \rightarrow 3 \pi$ is simulated at leading order (LO) in perturbative QCD using the Monte Carlo (MC) event generator PYTHIA 8.212 [12]. The transverse momentum spectrum of the $W$ boson $\left[p_{T}(W)\right.$ spectrum] is corrected to match that obtained in a $W \rightarrow \ell \nu(\ell=e, \mu$, $\tau)$ simulation in MADGRAPH5_aMC@NLO v2.3.3 [13] using the jet matching and merging scheme simulated at nextto-LO (NLO) through the FxFx algorithm [14]. The $Z / \gamma^{(*)} \rightarrow \ell \ell$ background is generated at LO using MADGRAPH5_aMC@NLO with the MLM jet matching algorithm [15]. Small background contributions from $W W$, $W Z$, and $Z Z$ (diboson) production are simulated at NLO with MADGRAPH5_aMC@NLO, whereas the $t \bar{t}$ process is simulated with the POWHEG 2.0 [16-20] generator. The generators are interfaced to PYTHIA to model parton showering and fragmentation. The PYTHIA parameters affecting the description of the underlying event are set to the CUETP8M1 tune [21]. The NNPDF 3.0 [22] parton distribution functions (PDF), with the order matching that used in the MC generation, are used in the simulation of all processes. Generated events are processed through a simulation of the CMS detector based on GEANT4 [23], and are reconstructed with the same algorithms used for 
data. Additional $p p$ collisions in the same or nearby bunch crossings (pileup) are simulated, and reweighted to reproduce the pileup multiplicity measured in data.

The reconstruction of events relies on the particle-flow (PF) algorithm [24], which combines information from the subdetectors to reconstruct and identify individual particles in an event. Combinations of these PF objects are used to reconstruct higher-level objects such as jets and hadronically decaying $\tau$ leptons, denoted as $\tau_{h}$. The reconstructed vertex with the largest value of summed physics-object $p_{T}{ }^{2}$ is the primary $p p$ interaction vertex. The physics objects are the jets, clustered using a jet-finding algorithm $[25,26]$ with the tracks assigned to the vertex as inputs, and the associated missing transverse momentum is the negative vector sum of the $p_{T}$ of those jets. The jets are reconstructed from PF objects with the anti- $k_{T}$ algorithm [25] implemented in the FASTJET library [26,27], using a distance parameter of 0.4.

Charged pions are reconstructed using the hadrons-plusstrips (HPS) algorithm [8,9], which is primarily designed to reconstruct $\tau_{h}$ candidates. This allows us to align the offline object reconstruction with the requirements of the analysis trigger, which are based on the presence of two high- $p_{T} \tau_{h}$ candidates, and to use advanced discriminators developed for the HPS algorithm to reject quark and gluon jets, electrons, and muons. The HPS algorithm is seeded using jets, and combines tracks and energy depositions in the ECAL to form $\tau_{h}$ candidates. Three decay modes are reconstructed: 1-prong, 1-prong $+\pi^{0}$, and 3-prong [8,9]. The 1-prong decay mode of the HPS algorithm targets $\tau$ leptons decaying to a charged pion and a $\nu_{\tau}$. About $85 \%$ of $\tau$ leptons decaying to a charged pion and $\mathrm{a} \nu_{\tau}$, and $15 \%$ of $\tau$ leptons decaying to a charged pion, a neutral pion, and a $\nu_{\tau}$, are reconstructed as 1 -prong $\tau_{h}$ candidates. Charged-pion candidates are required to be reconstructed in the 1-prong decay mode of the algorithm. They need to be isolated, in that the sum of the $p_{T}$ of charged particles and photons with $p_{T}>0.5 \mathrm{GeV}$ reconstructed with the $\mathrm{PF}$ algorithm around the $\tau_{h}$ object within a cone of $\Delta R=\sqrt{(\Delta \eta)^{2}+(\Delta \phi)^{2}}=0.3$ is required to be less than $2 \mathrm{GeV}$, where $\phi$ denotes the azimuthal angle measured in radians. This isolation criterion has an efficiency above $90 \%$ for each pion. The pion candidates must also pass veto requirements for electrons and muons [8,9]. The discriminator against muons makes use of information from the muon detectors. The efficiency for true $\tau_{h}$ or pions is larger than $99 \%$, while the misidentification rate is about $3.5 \times 10^{-3}$ [9]. The discriminator against electrons uses various hadron calorimeter and ECAL variables. Its efficiency is about $80 \%$, with the misidentification rate of the order of $3 \times 10^{-3}$ [9]. Pions must meet the minimum requirement that the distance of closest approach to the primary vertex satisfies $\left|d_{z}\right|<0.01 \mathrm{~cm}$ along the beam direction, and $d_{x y}<0.01 \mathrm{~cm}$ in the transverse plane.

Events are selected using triggers that require the presence of two $\tau_{h}$ candidates with $p_{T}$ thresholds greater than 35 or $40 \mathrm{GeV}$, depending on the run. About $3 \%$ of the signal events have two pions with $p_{T}>35 \mathrm{GeV}$ at generator level. Offline, the two pion candidates that correspond to the trigger objects are required to be reconstructed in the 1-prong $\tau_{h}$ decay mode, and to have $p_{T}>35 \mathrm{GeV}$ (or $40 \mathrm{GeV}$, depending on the trigger) and $|\eta|<2$.1. In addition, a third pion candidate is required to be present in the event, and be reconstructed as a 1-prong $\tau_{h}$ candidate with $p_{T}>18 \mathrm{GeV}$ and $|\eta|<2.3$. The selected 1-prong $\tau_{h}$ candidates are required to be separated from each other by at least $\Delta R=0.3$, be isolated, and pass the discriminator against muons. They must not all have the same electric charge. At least two of the pions are also required to pass the discriminator against electrons. The reconstructed $p_{T}$ of the $W$ boson, computed as the vectorial sum of the $\overrightarrow{p_{T}}$ of the three pion candidates, is required to be greater than $40 \mathrm{GeV}$. This selection criterion has an efficiency greater than $90 \%$ for signal events passing the other criteria listed above, because the $W$ bosons must have sufficiently high $p_{T}$ to pass the trigger requirements, and rejects more than twothirds of the background events.

The background is dominated by standard model events composed of jets produced through the strong interaction, referred to as QCD multijet events. This component is estimated from data, using a control region (CR1) with events that contain at least one HPS-reconstructed pion candidate failing the isolation condition. Such events are reweighted using an extrapolation factor that depends on the invariant mass of the three pions, $m_{3 \pi}$, to obtain the QCD multijet contribution in the signal region (SR). The extrapolation factor is obtained from events complementary to the $\mathrm{SR}$, selected with $p_{T}(W)<40 \mathrm{GeV}$. The dependence on $m_{3 \pi}$ is parametrized with a linear function. Because of the poor statistical uncertainty in the region with $p_{T}(W)<40 \mathrm{GeV}$, the chosen linear dependence on mass suffices to parametrize the $m_{3 \pi}$ spectrum. The contribution to CR1 of processes other than QCD multijet events, in particular $Z / \gamma^{(*)} \rightarrow \ell \ell$ events, is estimated from simulations and subtracted.

The second largest background corresponds to $Z / \gamma^{(*)} \rightarrow$ $\ell \ell$ events. The contribution from $Z / \gamma^{(*)} \rightarrow \tau \tau$ events, with $\tau$ decays to one charged hadron and a neutrino, is dominant, but $Z / \gamma^{(*)} \rightarrow e e$ and $Z / \gamma^{(*)} \rightarrow \mu \mu$ events also enter the SR because electrons and muons can easily be wrongly reconstructed as isolated 1-prong $\tau_{h}$ candidates by the HPS algorithm. The normalization of the $Z / \gamma^{(*)} \rightarrow \ell \ell$ background is estimated from simulation using the cross section computed in FEWZ 3.1.b2 [28]. The $p_{T}$ of the $Z$ boson and the mass spectra found in the LO simulation are corrected to match the data using correction factors obtained from $Z \rightarrow \mu \mu$ events [29]. The $m_{3 \pi}$ distribution for $Z / \gamma^{(*)} \rightarrow \ell \ell$ background in the SR comes from another control region in data (CR2), similar to the SR, but with the exception that two of the pion candidates are required to fail the discriminator that rejects electrons misidentified as $\tau_{h}$ candidates. The CR2 is enriched in $Z / \gamma^{(*)} \rightarrow e e$ events, but 
the $m_{3 \pi}$ distribution has been verified to be compatible for all $Z / \gamma^{(*)} \rightarrow \ell \ell$ decays.

The yields of the diboson, single top quark, $t \bar{t} Z, t \bar{t} W$, and triboson backgrounds are small, and these processes are estimated from simulation. The normalization of the $t \bar{t}$ background is estimated from simulation, while the $m_{3 \pi}$ distribution for these events is taken from data in a different control region (CR3) where there is at least one jet identified as originating from a bottom quark [30], and where the pion candidates pass relaxed criteria that discriminate against muons and electrons.

The results are obtained by using a binned maximum likelihood fit to the $m_{3 \pi}$ distribution. The mass resolution for the signal process is about 7\%. Systematic uncertainties are treated as nuisance parameters in the fit. The statistical uncertainty of the collected data constitutes about $90 \%$ of the total uncertainty in the final results.

The uncertainty in the integrated luminosity, applied to the signal and to backgrounds estimated from simulation, amounts to 2.5\% in 2016 [31] and 2.3\% in 2017 [32]. Because of the limited size of the simulated samples in the $\mathrm{SR}$, and data in CR1, CR2, and CR3, statistical uncertainties in individual bins of the $m_{3 \pi}$ distributions are treated as Poissonian nuisance parameters for all processes.

The leading systematic uncertainty is related to the pion identification efficiency and amounts to $5 \%$ per pion. This corresponds to an uncertainty of $15 \%$ for an event with three pions. The magnitude of the uncertainty is determined through a dedicated measurement in $Z \rightarrow \tau_{\mu} \tau_{h}$ events, where the $\tau_{h}$ candidates are reconstructed in the 1-prong decay mode and $\tau_{\mu}$ denotes a $\tau$ lepton decaying to a muon and neutrinos. The uncertainty in the trigger efficiency is uncorrelated with the pion identification efficiency uncertainty and amounts to an additional $3.5 \%$ for each pion accepted by the trigger [9]. For pions with $35<p_{T}<40 \mathrm{GeV}$, the trigger efficiency uncertainty is inflated by $10 \%$ because this $p_{T}$ region corresponds to a turn-on of the trigger, where uncertainties in the energy scale of trigger objects lead to large uncertainties in the efficiency. The signal sample contains about $65 \%$ of the events that have both triggering pions with $p_{T}>40 \mathrm{GeV}$, and $30 \%$ that have one triggering pion with $35<p_{T}<40 \mathrm{GeV}$. The uncertainty in the pion energy scale is also measured in $Z \rightarrow \tau_{\mu} \tau_{h}$ events, and amounts to $1.2 \%$ [9]. It affects both the normalization and the $m_{3 \pi}$ distribution for simulated processes with pions or genuine $\tau_{h}$ leptons. All these uncertainties are uncorrelated between the 2016 and 2017 data.

The uncertainty in the QCD multijet background comes from two sources. The first source reflects the parametrization of the extrapolation factor as a function of $m_{3 \pi}$ for events with at least one nonisolated pion candidate in CR1. The uncertainty in the slope of the linear function is propagated to the $m_{3 \pi}$ spectrum and treated as an uncertainty in the dependence of the distribution. The second source is related to the background estimation method, which makes use of events where at least one pion candidate is not isolated. This uncertainty amounts to $30 \%$, and is also uncorrelated between the two data-taking periods. The magnitude of the uncertainty is estimated from the results of three studies. The first compares the default background estimate to that obtained from reweighting events where one of the pions fails the isolation condition but still has an isolation value below a weaker threshold as high as $6 \mathrm{GeV}$, resulting in a normalization difference up to $18 \%$. In the second study, the default background estimate is compared to that obtained when the extrapolation factor is parametrized as a function of the leading pion $p_{T}$ or of $p_{T}(W)$. These alternative parametrizations lead to variations up to $20 \%$ in the QCD multijet background normalization. And in a third study, the agreement between data and predicted backgrounds is verified in a dedicated control region (CR4), where at least one of the pion candidates fails the $d_{x y}$ or $\left|d_{z}\right|$ requirements. The difference between the predicted backgrounds and data amounts to $23 \%$ in CR4. The uncertainty related to the subtraction of simulated events in CR1 is negligible with respect to the statistical uncertainty in the $m_{3 \pi}$ distribution of the QCD multijet background.

The theoretical uncertainty in the yield of the $Z / \gamma^{(*)} \rightarrow$ $\ell \ell$ background is $2 \%$, as determined in FEWZ 3.1.b2 [28]. The number of simulated $Z / \gamma^{(*)} \rightarrow \ell \ell$ events selected in the SR is small, and a statistical uncertainty in the normalization must be included explicitly because a smoothed $m_{3 \pi}$ distribution from CR2 is used in the SR. The statistical uncertainty in the $Z / \gamma^{(*)} \rightarrow \ell \ell$ yield amounts to 30 (20)\% in 2016 (2017). The cross sections of the diboson production processes and their uncertainties arise from next-to-NLO computations made using MCFM (v8.0) [33]. The full next-to-NLO with next-to-next-toleading logarithmic order resummation of soft gluon corrections [34-39], performed with the TOP++ 2.0 program [40], is used to compute a $t \bar{t}$ production cross section equal to $832_{-45}^{+40} \mathrm{pb}$ using a top quark mass of $172.5 \mathrm{GeV}$.

The theoretical uncertainty in the yield of $W$ bosons is $4 \%$, as determined with FEWZ 3.1.b2 [41,42]. It includes PDF and scale uncertainties. An uncertainty in the reweighting of the PYTHIA signal sample to NLO as a function of the generated $p_{T}(W)$ is also included. It amounts to $5 \%$ and is dominated by statistical uncertainties. As verified in $Z \rightarrow \mu \mu$ events, the difference in the $p_{T}$ spectra of the vector bosons between data and NLO simulation is at the subpercent level, and is therefore neglected.

Combining both data-taking periods, the $m_{3 \pi}$ distribution is shown in Fig. 1. No significant excess above the expected background is observed. Upper limits at a 95\% CL are set on the branching fraction of the $W$ boson to three charged pions using the $\mathrm{CL}_{s}$ method [43-45] and are shown in Fig. 2. These are $1.66 \times 10^{-6}$ in the 2016 data, $1.36 \times 10^{-6}$ in the 2017 data. The combination of the two data sets leads to 


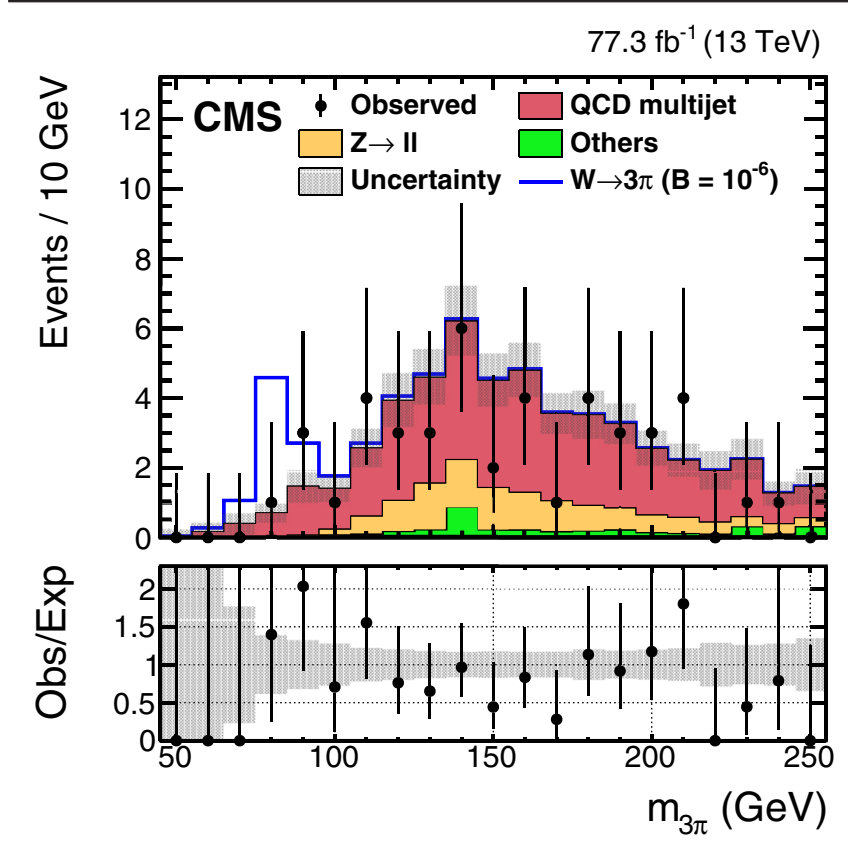

FIG. 1. Observed and expected distributions of $m_{3 \pi}$, using $77.3 \mathrm{fb}^{-1}$ of data collected in 2016 and 2017. The normalization of the expected backgrounds results from the maximum likelihood fit to the data, which includes all systematic uncertainties. The background contributions are stacked upon each other, and the "others" background contribution includes events from diboson, triboson, $t \bar{t}$, single top quark, $t \bar{t} Z$, and $t \bar{t} W$ production. The uncertainty band includes both statistical and systematic sources of uncertainty. The signal is normalized to $\mathcal{B}(W \rightarrow 3 \pi)=10^{-6}$, and is shown as an open stacked histogram.

$$
\mathcal{B}(W \rightarrow 3 \pi)<1.01 \times 10^{-6} .
$$

The expected sensitivity using the 2017 data is slightly worse than using the 2016 data despite the larger integrated luminosity because of higher $p_{T}$ thresholds to trigger the events during part of the run.

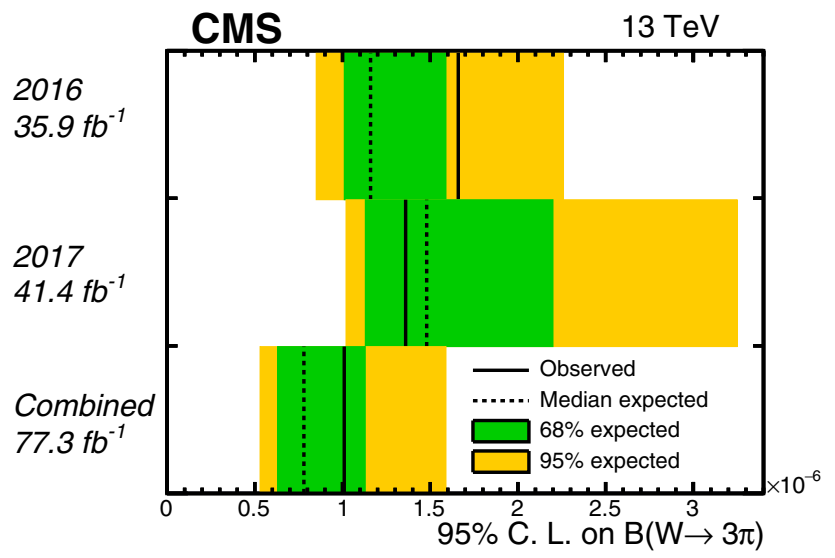

FIG. 2. Observed and expected upper limits on $\mathcal{B}(W \rightarrow 3 \pi)$ at $95 \%$ C. L. The expected limit corresponds to the assumption $\mathcal{B}(W \rightarrow 3 \pi)=0$.
In summary, the first search for the rare decay of the $W$ boson to three charged pions has been performed. The data correspond to an integrated luminosity of $77.3 \mathrm{fb}^{-1}$ of proton-proton collisions collected in the CMS detector at a center-of-mass energy of $13 \mathrm{TeV}$. Events with $W$ bosons are selected with a trigger designed to identify events with two $\tau$ leptons decaying to hadrons and a neutrino. The data are compatible with the background hypothesis. The upper limit on the branching fraction of the $W$ boson to three charged pions is $1.01 \times 10^{-6}$ at $95 \% \mathrm{CL}$, which reduces the previous range of values and motivates theoretical calculations of this branching fraction.

We congratulate our colleagues in the CERN accelerator departments for the excellent performance of the LHC and thank the technical and administrative staffs at CERN and at other CMS institutes for their contributions to the success of the CMS effort. In addition, we gratefully acknowledge the computing centers and personnel of the Worldwide LHC Computing Grid for delivering so effectively the computing infrastructure essential to our analyses. Finally, we acknowledge the enduring support for the construction and operation of the LHC and the CMS detector provided by the following funding agencies: BMBWF and FWF (Austria); FNRS and FWO (Belgium); CNPq, CAPES, FAPERJ, FAPERGS, and FAPESP (Brazil); MES (Bulgaria); CERN; CAS, MoST, and NSFC (China); COLCIENCIAS (Colombia); MSES and CSF (Croatia); RPF (Cyprus); SENESCYT (Ecuador); MoER, ERC IUT, and ERDF (Estonia); Academy of Finland, MEC, and HIP (Finland); CEA and CNRS/IN2P3 (France); BMBF, DFG, and HGF (Germany); GSRT (Greece); NKFIA (Hungary); DAE and DST (India); IPM (Iran); SFI (Ireland); INFN (Italy); MSIP and NRF (Republic of Korea); MES (Latvia); LAS (Lithuania); MOE and UM (Malaysia); BUAP, CINVESTAV, CONACYT, LNS, SEP, and UASLP-FAI (Mexico); MOS (Montenegro); MBIE (New Zealand); PAEC (Pakistan); MSHE and NSC (Poland); FCT (Portugal); JINR (Dubna); MON, RosAtom, RAS, RFBR, and NRC KI (Russia); MESTD (Serbia); SEIDI, CPAN, PCTI, and FEDER (Spain); MOSTR (Sri Lanka); Swiss Funding Agencies (Switzerland); MST (Taipei); ThEPCenter, IPST, STAR, and NSTDA (Thailand); TUBITAK and TAEK (Turkey); NASU and SFFR (Ukraine); STFC (United Kingdom); DOE and NSF (U.S.).

[1] Y. Grossman, K. Matthias, and M. Neubert, Exclusive radiative decays of $W$ and $Z$ bosons in QCD factorization, J. High Energy Phys. 04 (2015) 101.

[2] T. Melia, Exclusive hadronic W decay: $W \rightarrow \pi \gamma$ and $W \rightarrow \pi^{+} \pi^{+} \pi^{-}$, Nucl. Part. Phys. Proc. 273-275, 2102 (2016).

[3] G. Perez, Y. Soreq, E. Stamou, and K. Tobioka, Prospects for measuring the Higgs boson coupling to light quarks, Phys. Rev. D 93, 013001 (2016). 
[4] F. Abe et al. (CDF Collaboration), Search for the rare decay $W^{ \pm} \rightarrow D_{s}^{+-} \gamma$ in $p \bar{p}$ collisions at $\sqrt{s}=1.8 \mathrm{TeV}$, Phys. Rev. D 58, 091101 (1998).

[5] T. Aaltonen et al. (CDF Collaboration), Search for the rare radiative decay: $W \rightarrow \pi \gamma$ in $p \bar{p}$ collisions at $\sqrt{s}=1.96 \mathrm{TeV}$, Phys. Rev. D 85, 032001 (2012).

[6] M. Tanabashi et al. (Particle Data Group), Review of particle physics, Phys. Rev. D 98, 030001 (2018).

[7] M. Mangano and T. Melia, Rare exclusive hadronic $W$ decays in a $t \bar{t}$ environment, Eur. Phys. J. C 75, 258 (2015).

[8] CMS Collaboration, Reconstruction and identification of $\tau$ lepton decays to hadrons and $\nu_{\tau}$ at CMS, J. Instrum. 11, P01019 (2016).

[9] CMS Collaboration, Performance of reconstruction and identification of $\tau$ leptons decaying to hadrons and $\nu_{\tau}$ in $p p$ collisions at $\sqrt{s}=13 \mathrm{TeV}$, J. Instrum. 13, P10005 (2018).

[10] CMS Collaboration, The CMS trigger system, J. Instrum. 12, P01020 (2017).

[11] CMS Collaboration, The CMS experiment at the CERN LHC, J. Instrum. 3, S08004 (2008).

[12] T. Sjöstrand, S. Ask, J. R. Christiansen, R. Corke, N. Desai, P. Ilten, S. Mrenna, S. Prestel, C. O. Rasmussen, and P.Z. Skands, An introduction to PYTHIA 8.2, Comput. Phys. Commun. 191, 159 (2015).

[13] J. Alwall, R. Frederix, S. Frixione, V. Hirschi, F. Maltoni, O. Mattelaer, H. S. Shao, T. Stelzer, P. Torrielli, and M. Zaro, The automated computation of tree-level and next-toleading order differential cross sections, and their matching to parton shower simulations, J. High Energy Phys. 07 (2014) 079.

[14] R. Frederix and S. Frixione, Merging meets matching in MC@NLO, J. High Energy Phys. 12 (2012) 061.

[15] J. Alwall, S. Höche, F. Krauss, N. Lavesson, L. Lönnblad, F. Maltoni, M. L. Mangano, M. Moretti, C. G. Papadopoulos, F. Piccinini, S. Schumann, M. Treccani, J. Winter, and M. Worek, Comparative study of various algorithms for the merging of parton showers and matrix elements in hadronic collisions, Eur. Phys. J. C 53, 473 (2008).

[16] P. Nason, A new method for combining NLO QCD with shower Monte Carlo algorithms, J. High Energy Phys. 11 (2004) 040.

[17] S. Frixione, P. Nason, and C. Oleari, Matching NLO QCD computations with parton shower simulations: The POWHEG method, J. High Energy Phys. 11 (2007) 070.

[18] S. Alioli, P. Nason, C. Oleari, and E. Re, NLO Higgs boson production via gluon fusion matched with shower in POWHEG, J. High Energy Phys. 04 (2009) 002.

[19] S. Alioli, P. Nason, C. Oleari, and E. Re, A general framework for implementing NLO calculations in shower Monte Carlo programs: the POWHEG BOX, J. High Energy Phys. 06 (2010) 043.

[20] S. Alioli, K. Hamilton, P. Nason, C. Oleari, and E. Re, Jet pair production in POWHEG, J. High Energy Phys. 04 (2011) 081.

[21] CMS Collaboration, Event generator tunes obtained from underlying event and multiparton scattering measurements, Eur. Phys. J. C 76, 155 (2016).

[22] R. D. Ball et al. (NNPDF Collaboration), Parton distributions for the LHC Run II, J. High Energy Phys. 04 (2015) 040 .
[23] S. Agostinelli et al. (GEANT4 Collaboration), GEANT4A simulation toolkit, Nucl. Instrum. Methods Phys. Res., Sect. A 506, 250 (2003).

[24] CMS Collaboration, Particle-flow reconstruction and global event description with the CMS detector, J. Instrum. 12, P10003 (2017).

[25] M. Cacciari, G. P. Salam, and G. Soyez, The anti- $k_{T}$ jet clustering algorithm, J. High Energy Phys. 04 (2008) 063.

[26] M. Cacciari, G. P. Salam, and G. Soyez, FastJet user manual, Eur. Phys. J. C 72, 1896 (2012).

[27] M. Cacciari and G. P. Salam, Dispelling the $N^{3}$ myth for the $k_{T}$ jet-finder, Phys. Lett. B 641, 57 (2006).

[28] Y. Li and F. Petriello, Combining QCD and electroweak corrections to dilepton production in FEWZ, Phys. Rev. D 86, 094034 (2012).

[29] CMS Collaboration, Observation of the Higgs boson decay to a pair of $\tau$ leptons with the CMS detector, Phys. Lett. B 779, 283 (2018).

[30] CMS Collaboration, Identification of heavy-flavour jets with the CMS detector in $p p$ collisions at $13 \mathrm{TeV}$, J. Instrum. 13, P05011 (2018).

[31] CMS Collaboration, CMS Luminosity Measurements for the 2016 Data Taking Period, CERN, Geneva, Switzerland, CMS Physics Analysis Summary Report No. CMS-PASLUM-17-001, 2017, https://cds.cern.ch/record/2257069.

[32] CMS Collaboration, CMS luminosity measurement for the 2017 data-taking period at $\sqrt{s}=13 \mathrm{TeV}, \mathrm{CERN}$, Geneva, Switzerland, CMS Physics Analysis Summary Report No. CMS-PAS-LUM-17-004, 2018, https://cds.cern.ch/ record/2621960.

[33] J. M. Campbell and R. K. Ellis, MCFM for the Tevatron and the LHC, Nucl. Phys. B, Proc. Suppl. 205-206, 10 (2010).

[34] M. Beneke, P. Falgari, S. Klein, and C. Schwinn, Hadronic top quark pair production with NNLL threshold resummation, Nucl. Phys. B855, 695 (2012).

[35] M. Cacciari, M. Czakon, M. Mangano, A. Mitov, and P. Nason, Top pair production at hadron colliders with next-tonext-to-leading logarithmic soft-gluon resummation, Phys. Lett. B 710, 612 (2012).

[36] P. Bärnreuther, M. Czakon, and A. Mitov, Percent Level Precision Physics at the Tevatron: First Genuine NNLO QCD Corrections to $q \bar{q} \rightarrow t \bar{t}+X$, Phys. Rev. Lett. 109, 132001 (2012).

[37] M. Czakon and A. Mitov, NNLO corrections to top pair production at hadron colliders: The quark-gluon reaction, J. High Energy Phys. 01 (2013) 080.

[38] M. Czakon and A. Mitov, NNLO corrections to top pair production at hadron colliders: the all-fermionic scattering channels, J. High Energy Phys. 12 (2012) 054.

[39] M. Czakon, P. Fiedler, and A. Mitov, Total Top Quark Pair Production Cross Section at Hadron Colliders Through $\mathrm{O}\left(\alpha_{s}^{4}\right)$, Phys. Rev. Lett. 110, 252004 (2013).

[40] M. Czakon and A. Mitov, Top++: A program for the calculation of the top pair cross section at hadron colliders, Comput. Phys. Commun. 185, 2930 (2014).

[41] R. Gavin, Y. Li, F. Petriello, and S. Quackenbush, W physics at the LHC with FEWZ 2.1, Comput. Phys. Commun. 184, 209 (2013). 
[42] K. Melnikov and F. Petriello, Electroweak gauge boson production at hadron colliders through $O\left(\alpha_{S}^{2}\right)$, Phys. Rev. D 74, 114017 (2006).

[43] ATLAS and CMS Collaborations, LHC Higgs Combination Group, Procedure for the LHC Higgs boson search combination in Summer 2011, CERN, Geneva, Switzerland, Technical Report Nos. ATL-PHYS-PUB
2011-11, CMS NOTE 2011/005, 2011, https://cds.cern .ch/record/1379837.

[44] T. Junk, Confidence level computation for combining searches with small statistics, Nucl. Instrum. Methods Phys. Res., Sect. A 434, 435 (1999).

[45] A. L. Read, Presentation of search results: The $\mathrm{CL}_{s}$ technique, J. Phys. G 28, 2693 (2002).

A. M. Sirunyan, ${ }^{1}$ A. Tumasyan, ${ }^{1}$ W. Adam, ${ }^{2}$ F. Ambrogi, ${ }^{2}$ E. Asilar, ${ }^{2}$ T. Bergauer, ${ }^{2}$ J. Brandstetter, ${ }^{2}$ M. Dragicevic, ${ }^{2}$ J. Erö, ${ }^{2}$ A. Escalante Del Valle, ${ }^{2}$ M. Flechl, ${ }^{2}$ R. Frühwirth, ${ }^{2, b}$ V. M. Ghete, ${ }^{2}$ J. Hrubec, ${ }^{2}$ M. Jeitler, ${ }^{2, b}$ N. Krammer, ${ }^{2}$ I. Krätschmer, ${ }^{2}$ D. Liko, ${ }^{2}$ T. Madlener, ${ }^{2}$ I. Mikulec, ${ }^{2}$ N. Rad, ${ }^{2}$ H. Rohringer, ${ }^{2}$ J. Schieck, ${ }^{2, b}$ R. Schöfbeck, ${ }^{2}$ M. Spanring, ${ }^{2}$ D. Spitzbart, ${ }^{2}$ W. Waltenberger, ${ }^{2}$ J. Wittmann, ${ }^{2}$ C.-E. Wulz, ${ }^{2, b}$ M. Zarucki, ${ }^{2}$ V. Chekhovsky, ${ }^{3}$ V. Mossolov, ${ }^{3}$ J. Suarez Gonzalez, ${ }^{3}$ E. A. De Wolf, ${ }^{4}$ D. Di Croce, ${ }^{4}$ X. Janssen, ${ }^{4}$ J. Lauwers, ${ }^{4}$ A. Lelek, ${ }^{4}$ M. Pieters ${ }^{4}$ H. Van Haevermaet, ${ }^{4}$ P. Van Mechelen, ${ }^{4}$ N. Van Remortel, ${ }^{4}$ F. Blekman, ${ }^{5}$ J. D'Hondt, ${ }^{5}$ J. De Clercq, ${ }^{5}$ K. Deroover, ${ }^{5}$ G. Flouris, ${ }^{5}$ D. Lontkovskyi, ${ }^{5}$ S. Lowette, ${ }^{5}$ I. Marchesini, ${ }^{5}$ S. Moortgat, ${ }^{5}$ L. Moreels, ${ }^{5}$ Q. Python, ${ }^{5}$ K. Skovpen, ${ }^{5}$ S. Tavernier, ${ }^{5}$ W. Van Doninck, ${ }^{5}$ P. Van Mulders, ${ }^{5}$ I. Van Parijs, ${ }^{5}$ D. Beghin, ${ }^{6}$ B. Bilin, ${ }^{6}$ H. Brun, ${ }^{6}$ B. Clerbaux,${ }^{6}$ G. De Lentdecker, ${ }^{6}$ H. Delannoy, ${ }^{6}$ B. Dorney, ${ }_{6}^{6}$ G. Fasanella, ${ }^{6}$ L. Favart, ${ }^{6}$ A. Grebenyuk, ${ }^{6}$ A. K. Kalsi, ${ }^{6}$ J. Luetic, ${ }^{6}$ A. Popov, ${ }^{6, c}$ N. Postiau, ${ }^{6}$ E. Starling, ${ }^{6}$ L. Thomas, ${ }^{6}$ C. Vander Velde, ${ }^{6}$ P. Vanlaer, ${ }^{6}$ D. Vannerom, ${ }^{6}$ Q. Wang, ${ }^{6}$ T. Cornelis, ${ }^{7}$ D. Dobur, ${ }^{7}$ A. Fagot, ${ }^{7}$ M. Gul, ${ }^{7}$ I. Khvastunov, ${ }^{7, d}$ C. Roskas, ${ }^{7}$ D. Trocino, ${ }^{7}$ M. Tytgat, ${ }^{7}$ W. Verbeke, ${ }^{7}$ B. Vermassen, ${ }^{7}$ M. Vit, ${ }^{7}$ N. Zaganidis, ${ }^{7}$ O. Bondu, ${ }^{8}$ G. Bruno, ${ }^{8}$ C. Caputo, ${ }^{8}$ P. David, ${ }^{8}$ C. Delaere, ${ }^{8}$ M. Delcourt, ${ }^{8}$ A. Giammanco,${ }^{8}$ G. Krintiras,${ }^{8}$ V. Lemaitre,${ }^{8}$ A. Magitteri, ${ }^{8}$ K. Piotrzkowski, ${ }^{8}$ A. Saggio, ${ }^{8}$

M. Vidal Marono, ${ }^{8}$ P. Vischia, ${ }^{8}$ J. Zobec,${ }^{8}$ F. L. Alves,${ }^{9}$ G. A. Alves, ${ }^{9}$ G. Correia Silva, ${ }^{9}$ C. Hensel, ${ }^{9}$ A. Moraes, ${ }^{9}$ M. E. Pol, ${ }^{9}$ P. Rebello Teles, ${ }^{9}$ E. Belchior Batista Das Chagas, ${ }^{10}$ W. Carvalho, ${ }^{10}$ J. Chinellato, ${ }^{10, e}$ E. Coelho, ${ }^{10}$ E. M. Da Costa, ${ }^{10}$ G. G. Da Silveira, ${ }^{10, \mathrm{f}}$ D. De Jesus Damiao, ${ }^{10}$ C. De Oliveira Martins, ${ }^{10}$ S. Fonseca De Souza, ${ }^{10}$ L. M. Huertas Guativa, ${ }^{10}$ H. Malbouisson, ${ }^{10}$ D. Matos Figueiredo, ${ }^{10}$ M. Melo De Almeida, ${ }^{10}$ C. Mora Herrera,${ }^{10}$ L. Mundim, ${ }^{10}$ H. Nogima, ${ }^{10}$ W. L. Prado Da Silva, ${ }^{10}$ L. J. Sanchez Rosas, ${ }^{10}$ A. Santoro, ${ }^{10}$ A. Sznajder,${ }^{10}$ M. Thiel,${ }^{10}$ E. J. Tonelli Manganote,${ }^{10, e}$ F. Torres Da Silva De Araujo, ${ }^{10}$ A. Vilela Pereira, ${ }^{10}$ S. Ahuja, ${ }^{11 a}$ C. A. Bernardes, ${ }^{11 a}$ L. Calligaris,${ }^{11 a}$

T. R. Fernandez Perez Tomei, ${ }^{11 \mathrm{a}}$ E. M. Gregores, ${ }^{11 \mathrm{a}, 1 \mathrm{bb}}$ P. G. Mercadante, ${ }^{11 \mathrm{a}, 11 \mathrm{~b}}$ S. F. Novaes, ${ }^{11 \mathrm{a}}$ Sandra S. Padula, ${ }^{11 \mathrm{a}}$ A. Aleksandrov, ${ }^{12}$ R. Hadjiiska, ${ }^{12}$ P. Iaydjiev, ${ }^{12}$ A. Marinov, ${ }^{12}$ M. Misheva, ${ }^{12}$ M. Rodozov, ${ }^{12}$ M. Shopova, ${ }^{12}$ G. Sultanov, ${ }^{12}$ A. Dimitrov ${ }^{13}$ L. Litov, ${ }^{13}$ B. Pavlov,${ }^{13}$ P. Petkov, ${ }^{13}$ W. Fang, ${ }^{14, \mathrm{~g}}$ X. Gao,${ }^{14, \mathrm{~g}}$ L. Yuan, ${ }^{14}$ M. Ahmad, ${ }^{15}$ J. G. Bian, ${ }^{15}$ G. M. Chen, ${ }^{15}$ H. S. Chen, ${ }^{15}$ M. Chen, ${ }^{15}$ Y. Chen, ${ }^{15}$ C. H. Jiang, ${ }^{15}$ D. Leggat, ${ }^{15}$ H. Liao, ${ }^{15}$ Z. Liu, ${ }^{15}$ S. M. Shaheen, ${ }^{15, h}$ A. Spiezia, ${ }^{15}$ J. Tao, ${ }^{15}$ E. Yazgan,${ }^{15}$ H. Zhang, ${ }^{15}$ S. Zhang, ${ }^{15, h}$ J. Zhao, ${ }^{15}$ Y. Ban, ${ }^{16}$ G. Chen, ${ }^{16}$ A. Levin, ${ }^{16}$ J. Li, ${ }^{16}$ L. Li, ${ }^{16}$ Q. Li, ${ }^{16}$ Y. Mao, ${ }^{16}$ S. J. Qian, ${ }^{16}$ D. Wang, ${ }^{16}$ Y. Wang,,${ }^{17}$ C. Avila, ${ }^{18}$ A. Cabrera,${ }^{18}$ C. A. Carrillo Montoya,${ }^{18}$

L. F. Chaparro Sierra, ${ }^{18}$ C. Florez ${ }^{18}$ C. F. González Hernández, ${ }^{18}$ M. A. Segura Delgado, ${ }^{18}$ J. D. Ruiz Alvarez, ${ }^{19}$ N. Godinovic ${ }^{20}$ D. Lelas,${ }^{20}$ I. Puljak, ${ }^{20}$ T. Sculac ${ }^{20}$ Z. Antunovic, ${ }^{21}$ M. Kovac,${ }^{21}$ V. Brigljevic, ${ }^{22}$ D. Ferencek, ${ }^{22}$ K. Kadija, ${ }^{22}$ B. Mesic, ${ }^{22}$ M. Roguljic, ${ }^{22}$ A. Starodumov ${ }^{22, i}$ T. Susa,${ }^{22}$ M. W. Ather ${ }^{23}$ A. Attikis, ${ }^{23}$ M. Kolosova, ${ }^{23}$ G. Mavromanolakis,${ }^{23}$ J. Mousa, ${ }^{23}$ C. Nicolaou, ${ }^{23}$ F. Ptochos,${ }^{23}$ P. A. Razis, ${ }^{23}$ H. Rykaczewski, ${ }^{23}$ M. Finger, ${ }^{24, j}$ M. Finger Jr., ${ }^{24, j}$ E. Ayala, ${ }^{25}$ E. Carrera Jarrin, ${ }^{26}$ A. Ellithi Kamel, ${ }^{27, k}$ M. A. Mahmoud, ${ }^{27,1, \mathrm{~m}}$ E. Salama, ${ }^{27, \mathrm{n}, 1}$ S. Bhowmik, ${ }^{28}$

A. Carvalho Antunes De Oliveira, ${ }^{28}$ R. K. Dewanjee, ${ }^{28}$ K. Ehataht, ${ }^{28}$ M. Kadastik, ${ }^{28}$ M. Raidal,,${ }^{28}$ C. Veelken, ${ }^{28}$ P. Eerola, ${ }^{29}$ H. Kirschenmann, ${ }^{29}$ J. Pekkanen, ${ }^{29}$ M. Voutilainen, ${ }^{29}$ J. Havukainen, ${ }^{30}$ J. K. Heikkilä, ${ }^{30}$ T. Järvinen,${ }^{30}$ V. Karimäki,${ }^{30}$ R. Kinnunen, ${ }^{30}$ T. Lampén, ${ }^{30}$ K. Lassila-Perini, ${ }^{30}$ S. Laurila ${ }^{30}$ S. Lehti, ${ }^{30}$ T. Lindén, ${ }^{30}$ P. Luukka, ${ }^{30}$ T. Mäenpää, ${ }^{30}$ H. Siikonen, ${ }^{30}$ E. Tuominen, ${ }^{30}$ J. Tuominiemi, ${ }^{30}$ T. Tuuva, ${ }^{31}$ M. Besancon, ${ }^{32}$ F. Couderc, ${ }^{32}$ M. Dejardin, ${ }^{32}$ D. Denegri, ${ }^{32}$ J. L. Faure, ${ }^{32}$ F. Ferri, ${ }^{32}$ S. Ganjour, ${ }^{32}$ A. Givernaud ${ }^{32}$ P. Gras, ${ }^{32}$ G. Hamel de Monchenault, ${ }^{32}$ P. Jarry, ${ }^{32}$ C. Leloup ${ }^{32}$ E. Locci, ${ }^{32}$ J. Malcles, ${ }^{32}$ J. Rander, ${ }^{32}$ A. Rosowsky, ${ }^{32}$ M. Ö. Sahin, ${ }^{32}$ A. Savoy-Navarro, ${ }^{32,0}$ M. Titov, ${ }^{32}$ C. Amendola, ${ }^{33}$ F. Beaudette, ${ }^{33}$ P. Busson, ${ }^{33}$ C. Charlot ${ }^{33}$ B. Diab, ${ }^{33}$ R. Granier de Cassagnac, ${ }^{33}$ I. Kucher, ${ }^{33}$ A. Lobanov, ${ }^{33}$ J. Martin Blanco, ${ }^{33}$ C. Martin Perez,${ }^{33}$ M. Nguyen, ${ }^{33}$ C. Ochando, ${ }^{33}$ G. Ortona,${ }^{33}$ P. Paganini, ${ }^{33}$ J. Rembser, ${ }^{33}$ R. Salerno, ${ }^{33}$ J. B. Sauvan, ${ }^{33}$ Y. Sirois, ${ }^{33}$ A. Zabi, ${ }^{33}$ A. Zghiche, ${ }^{33}$ J.-L. Agram, ${ }^{34, p}$ J. Andrea, ${ }^{34}$ D. Bloch, ${ }^{34}$ G. Bourgatte, ${ }^{34}$ J.-M. Brom, ${ }^{34}$ E. C. Chabert, ${ }^{34}$ V. Cherepanov,${ }^{34}$ C. Collard ${ }^{34}$ E. Conte, ${ }^{34, p}$ J.-C. Fontaine,${ }^{34, p}$ D. Gelé, ${ }^{34}$ U. Goerlach,${ }^{34}$ M. Jansová, ${ }^{34}$ A.-C. Le Bihan, ${ }^{34}$ N. Tonon, ${ }^{34}$ P. Van Hove, ${ }^{34}$ S. Gadrat,${ }^{35}$ S. Beauceron, ${ }^{36}$ C. Bernet, ${ }^{36}$ G. Boudoul, ${ }^{36}$ N. Chanon, ${ }^{36}$ 
R. Chierici, ${ }^{36}$ D. Contardo, ${ }^{36}$ P. Depasse, ${ }^{36}$ H. El Mamouni, ${ }^{36}$ J. Fay, ${ }^{36}$ S. Gascon,${ }^{36}$ M. Gouzevitch,${ }^{36}$ G. Grenier, ${ }^{36}$ B. Ille ${ }^{36}$ F. Lagarde ${ }^{36}$ I. B. Laktineh ${ }^{36}$ H. Lattaud ${ }^{36}$ M. Lethuillier, ${ }^{36}$ L. Mirabito, ${ }^{36}$ S. Perries,${ }^{36}$ V. Sordini, ${ }^{36}$ G. Touquet, ${ }^{36}$ M. Vander Donckt, ${ }^{36}$ S. Viret ${ }^{36}$ T. Toriashvilii, ${ }^{37, q}$ Z. Tsamalaidze, ${ }^{38, j}$ C. Autermann, ${ }^{39}$ L. Feld,${ }^{39}$ M. K. Kiesel, ${ }^{39}$ K. Klein, ${ }^{39}$ M. Lipinski, ${ }^{39}$ M. Preuten, ${ }^{39}$ M. P. Rauch, ${ }^{39}$ C. Schomakers,${ }^{39}$ J. Schulz, ${ }^{39}$ M. Teroerde,${ }^{39}$ B. Wittmer, ${ }^{39}$ A. Albert, ${ }^{40}$ M. Erdmann, ${ }^{40}$ S. Erdweg, ${ }^{40}$ T. Esch, ${ }^{40}$ R. Fischer, ${ }^{40}$ S. Ghosh,${ }^{40}$ T. Hebbeker, ${ }^{40}$ C. Heidemann, ${ }^{40}$ K. Hoepfner,${ }^{40}$ H. Keller, ${ }^{40}$ L. Mastrolorenzo, ${ }^{40}$ M. Merschmeyer, ${ }^{40}$ A. Meyer, ${ }^{40}$ P. Millet,${ }^{40}$ S. Mukherjee, ${ }^{40}$ A. Novak ${ }^{40}$ T. Pook,${ }^{40}$ A. Pozdnyakov,${ }^{40}$ M. Radziej, ${ }^{40}$ H. Reithler, ${ }^{40}$ M. Rieger, ${ }^{40}$ A. Schmidt, ${ }^{40}$ A. Sharma ${ }^{40}$ D. Teyssier, ${ }^{40}$ S. Thüer, ${ }^{40}$ G. Flügge, ${ }^{41}$ O. Hlushchenko, ${ }^{41}$ T. Kress, ${ }^{41}$ T. Müller, ${ }^{41}$ A. Nehrkorn, ${ }^{41}$ A. Nowack, ${ }^{41}$ C. Pistone, ${ }^{41}$ O. Pooth, ${ }^{41}$ D. Roy, ${ }^{41}$ H. Sert, ${ }^{41}$ A. Stahl ${ }^{41, r}$ M. Aldaya Martin, ${ }^{42}$ T. Arndt, ${ }^{42}$ C. Asawatangtrakuldee, ${ }^{42}$ I. Babounikau, ${ }^{42}$ H. Bakhshiansohi, ${ }^{42}$ K. Beernaert ${ }^{42}$ O. Behnke, ${ }^{42}$ U. Behrens, ${ }^{42}$ A. Bermúdez Martínez ${ }^{42}$ D. Bertsche, ${ }^{42}$ A. A. Bin Anuar, ${ }^{42}$ K. Borras, ${ }^{42, s}$ V. Botta, ${ }^{42}$ A. Campbell, ${ }^{42}$ P. Connor, ${ }^{42}$ C. Contreras-Campana, ${ }^{42}$ V. Danilov, ${ }^{42}$ A. De Wit, ${ }^{42}$ M. M. Defranchis, ${ }^{42}$ C. Diez Pardos, ${ }^{42}$ D. Domínguez Damiani ${ }^{42}$ G. Eckerlin, ${ }^{42}$ T. Eichhorn, ${ }^{42}$ A. Elwood, ${ }^{42}$ E. Eren ${ }^{42}$ E. Gallo, ${ }^{42, t}$ A. Geiser, ${ }^{42}$ J. M. Grados Luyando, ${ }^{42}$ A. Grohsjean, ${ }^{42}$ M. Guthoff, ${ }^{42}$ M. Haranko, ${ }^{42}$ A. Harb, ${ }^{42}$ N. Z. Jomhari, ${ }^{42}$ H. Jung, ${ }^{42}$ M. Kasemann, ${ }^{42}$ J. Keaveney, ${ }^{42}$ C. Kleinwort, ${ }^{42}$ J. Knolle, ${ }^{42}$ D. Krücker, ${ }^{42}$ W. Lange, ${ }^{42}$ T. Lenz, ${ }^{42}$ J. Leonard, ${ }^{42}$ K. Lipka, ${ }^{42}$ W. Lohmann, ${ }^{42, \mathrm{u}}$ R. Mankel, ${ }^{42}$ I.-A. Melzer-Pellmann, ${ }^{42}$ A. B. Meyer, ${ }^{42}$ M. Meyer, ${ }^{42}$ M. Missiroli, ${ }^{42}$ G. Mittag, ${ }^{42}$ J. Mnich ${ }^{42}$ V. Myronenko, ${ }^{42}$ S. K. Pflitsch, ${ }^{42}$ D. Pitzl, ${ }^{42}$ A. Raspereza, ${ }^{42}$ A. Saibel ${ }^{42}$ M. Savitskyi, ${ }^{42}$ P. Saxena, ${ }^{42}$ P. Schütze, ${ }^{42}$ C. Schwanenberger, ${ }^{42}$ R. Shevchenko, ${ }^{42}$ A. Singh ${ }^{42}$ H. Tholen, ${ }^{42}$ O. Turkot, ${ }^{42}$ A. Vagnerini, ${ }^{42}$ M. Van De Klundert, ${ }^{42}$ G. P. Van Onsem, ${ }^{42}$ R. Walsh, ${ }^{42}$ Y. Wen, ${ }^{42}$ K. Wichmann,${ }^{42}$ C. Wissing, ${ }^{42}$ O. Zenaiev, ${ }^{42}$ R. Aggleton, ${ }^{43}$ S. Bein, ${ }^{43}$ L. Benato, ${ }^{43}$ A. Benecke, ${ }^{43}$ V. Blobel,${ }^{43}$ T. Dreyer ${ }^{43}$ A. Ebrahimi,${ }^{43}$ E. Garutti, ${ }^{43}$ D. Gonzalez,${ }^{43}$ P. Gunnellini, ${ }^{43}$ J. Haller,${ }^{43}$ A. Hinzmann, ${ }^{43}$ A. Karavdina, ${ }^{43}$ G. Kasieczka, ${ }^{43}$ R. Klanner, ${ }^{43}$ R. Kogler, ${ }^{43}$ N. Kovalchuk, ${ }^{43}$ S. Kurz, ${ }^{43}$ V. Kutzner, ${ }^{43}$ J. Lange, ${ }^{43}$ D. Marconi, ${ }^{43}$ J. Multhaup, ${ }^{43}$ M. Niedziela, ${ }^{43}$ C. E. N. Niemeyer, ${ }^{43}$ D. Nowatschin, ${ }^{43}$ A. Perieanu, ${ }^{43}$

A. Reimers, ${ }^{43}$ O. Rieger, ${ }^{43}$ C. Scharf, ${ }^{43}$ P. Schleper ${ }^{43}$ S. Schumann, ${ }^{43}$ J. Schwandt, ${ }^{43}$ J. Sonneveld, ${ }^{43}$ H. Stadie, ${ }^{43}$ G. Steinbrück, ${ }^{43}$ F. M. Stober, ${ }^{43}$ M. Stöver, ${ }^{43}$ B. Vormwald, ${ }^{43}$ I. Zoi, ${ }^{43}$ M. Akbiyik, ${ }^{44}$ C. Barth, ${ }^{44}$ M. Baselga ${ }^{44}$ S. Baur,${ }^{44}$ T. Berger, ${ }^{44}$ E. Butz, ${ }^{44}$ R. Caspart, ${ }^{44}$ T. Chwalek, ${ }^{44}$ W. De Boer, ${ }^{44}$ A. Dierlamm, ${ }^{44}$ K. El Morabit, ${ }^{44}$ N. Faltermann, ${ }^{44}$ M. Giffels, ${ }^{44}$ M. A. Harrendorf, ${ }^{44}$ F. Hartmann, ${ }^{44, r}$ U. Husemann, ${ }^{44}$ I. Katkov, ${ }^{44, c}$ S. Kudella, ${ }^{44}$ S. Mitra, ${ }^{44}$ M. U. Mozer, ${ }^{44}$ Th. Müller, ${ }^{44}$ M. Musich, ${ }^{44}$ G. Quast ${ }^{44}$ K. Rabbertz ${ }^{44}$ M. Schröder, ${ }^{44}$ I. Shvetsov, ${ }^{44}$ H. J. Simonis, ${ }^{44}$ R. Ulrich, ${ }^{44}$ M. Weber, ${ }^{44}$ C. Wöhrmann,${ }^{44}$ R. Wolf, ${ }^{44}$ G. Anagnostou, ${ }^{45}$ G. Daskalakis, ${ }^{45}$ T. Geralis, ${ }^{45}$ A. Kyriakis, ${ }^{45}$ D. Loukas, ${ }^{45}$ G. Paspalaki, ${ }^{45}$ A. Agapitos, ${ }^{46}$ G. Karathanasis, ${ }^{46}$ P. Kontaxakis, ${ }^{46}$ A. Panagiotou, ${ }^{46}$ I. Papavergou, ${ }^{46}$ N. Saoulidou, ${ }^{46}$ K. Vellidis, ${ }^{46}$ G. Bakas, ${ }^{47}$ K. Kousouris,${ }^{47}$ I. Papakrivopoulos, ${ }^{47}$ G. Tsipolitis, ${ }^{47}$ I. Evangelou,${ }^{48}$ C. Foudas,${ }^{48}$ P. Gianneios,${ }^{48}$ P. Katsoulis, ${ }^{48}$ P. Kokkas, ${ }^{48}$ S. Mallios ${ }^{48}$ K. Manitara ${ }^{48}$ N. Manthos ${ }^{48}$ I. Papadopoulos ${ }^{48}$ E. Paradas ${ }^{48}$ J. Strologas, ${ }^{48}$ F. A. Triantis, ${ }^{48}$ D. Tsitsonis, ${ }^{48}$ M. Bartók, ${ }^{49, v}$ M. Csanad, ${ }^{49}$ N. Filipovic, ${ }^{49}$ P. Major, ${ }^{49}$ K. Mandal, ${ }^{49}$ A. Mehta, ${ }^{49}$ M. I. Nagy, ${ }^{49}$ G. Pasztor ${ }^{49}$ O. Surányi, ${ }^{49}$ G. I. Veres, ${ }^{49}$ G. Bencze,${ }^{50}$ C. Hajdu, ${ }^{50}$ D. Horvath, ${ }^{50, w}$ Á. Hunyadi, ${ }^{50}$ F. Sikler, ${ }^{50}$ T. Á. Vámi ${ }^{50}$ V. Veszpremi,${ }^{50}$ G. Vesztergombi,${ }^{50, a, x}$ N. Beni, ${ }^{51}$ S. Czellar, ${ }^{51}$ J. Karancsi ${ }^{51, v}$ A. Makovec,${ }^{51}$ J. Molnar,${ }^{51}$ Z. Szillasi,${ }^{51}$ P. Raics, ${ }^{52}$ Z. L. Trocsanyi, ${ }^{52}$ B. Ujvari, ${ }^{52}$ S. Choudhury, ${ }^{53}$ J. R. Komaragiri, ${ }^{53}$ P. C. Tiwari, ${ }^{53}$ S. Bahinipati, ${ }^{54, y}$ C. Kar, ${ }^{54}$ P. Mal, ${ }^{54}$ A. Nayak, ${ }^{54, z}$ S. Roy Chowdhury, ${ }^{54}$ D. K. Sahoo, ${ }^{54, y}$ S. K. Swain, ${ }^{54}$ S. Bansal, ${ }^{55}$ S. B. Beri, ${ }^{55}$ V. Bhatnagar, ${ }^{55}$ S. Chauhan, ${ }^{55}$ R. Chawla, ${ }^{55}$ N. Dhingra, ${ }^{55}$ R. Gupta, ${ }^{55}$ A. Kaur, ${ }^{55}$ M. Kaur, ${ }^{55}$ S. Kaur, ${ }^{55}$ P. Kumari, ${ }^{55}$ M. Lohan, ${ }^{55}$ M. Meena ${ }^{55}$ K. Sandeep,${ }^{55}$ S. Sharma,${ }^{55}$ J. B. Singh, ${ }^{55}$ A. K. Virdi,${ }^{55}$ G. Walia, ${ }^{55}$ A. Bhardwaj, ${ }^{56}$

B. C. Choudhary, ${ }^{56}$ R. B. Garg, ${ }^{56}$ M. Gola, ${ }^{56}$ S. Keshri, ${ }^{56}$ Ashok Kumar, ${ }^{56}$ S. Malhotra, ${ }^{56}$ M. Naimuddin, ${ }^{56}$ P. Priyanka ${ }^{56}$ K. Ranjan, ${ }^{56}$ Aashaq Shah, ${ }^{56}$ R. Sharma ${ }^{56}$ R. Bhardwaj, ${ }^{57, a a}$ M. Bharti, ${ }^{57, \text { aa }}$ R. Bhattacharya, ${ }^{57}$ S. Bhattacharya, ${ }^{57}$ U. Bhawandeep, ${ }^{57, \text { aa }}$ D. Bhowmik, ${ }^{57}$ S. Dey, ${ }^{57}$ S. Dutt, ${ }^{57, \text { aa }}$ S. Dutta, ${ }^{57}$ S. Ghosh,${ }^{57}$ M. Maity, ${ }^{57, b b}$ K. Mondal, ${ }^{57}$ S. Nandan, ${ }^{57}$ A. Purohit, ${ }^{57}$ P. K. Rout, ${ }^{57}$ A. Roy, ${ }^{57}$ G. Saha, ${ }^{57}$ S. Sarkar, ${ }^{57}$ T. Sarkar, ${ }^{57, b b}$ M. Sharan, ${ }^{57}$ B. Singh, ${ }^{57, \text { aa }}$ S. Thakur, ${ }^{57, \text { aa }}$ P. K. Behera, ${ }^{58}$ A. Muhammad, ${ }^{58}$ R. Chudasama, ${ }^{59}$ D. Dutta, ${ }^{59}$ V. Jha ${ }^{59}$ V. Kumar, ${ }^{59}$ D. K. Mishra, ${ }^{59}$ P. K. Netrakanti, ${ }^{59}$ L. M. Pant, ${ }^{59}$ P. Shukla, ${ }^{59}$ P. Suggisetti, ${ }^{59}$ T. Aziz, ${ }^{60}$ M. A. Bhat, ${ }^{60}$ S. Dugad, ${ }^{60}$ G. B. Mohanty, ${ }^{60}$ N. Sur,${ }^{60}$

Ravindra Kumar Verma ${ }^{60}$ S. Banerjee, ${ }^{61}$ S. Bhattacharya,${ }^{61}$ S. Chatterjee,${ }^{61}$ P. Das,${ }^{61}$ M. Guchait, ${ }^{61}$ Sa. Jain, ${ }^{61}$ S. Karmakar, ${ }^{61}$ S. Kumar, ${ }^{61}$ G. Majumder, ${ }^{61}$ K. Mazumdar, ${ }^{61}$ N. Sahoo, ${ }^{61}$ S. Sawant, ${ }^{61}$ S. Chauhan, ${ }^{62}$ S. Dube, ${ }^{62}$ V. Hegde, ${ }^{62}$ A. Kapoor, ${ }^{62}$ K. Kothekar, ${ }^{62}$ S. Pandey,${ }^{62}$ A. Rane, ${ }^{62}$ A. Rastogi ${ }^{62}$ S. Sharma ${ }^{62}$ S. Chenarani, ${ }^{63, c c}$ E. Eskandari Tadavani ${ }^{63}$ S. M. Etesami, ${ }^{63, \mathrm{cc}}$ M. Khakzad, ${ }^{63}$ M. Mohammadi Najafabadi, ${ }^{63}$ M. Naseri, ${ }^{63}$ F. Rezaei Hosseinabadi, ${ }^{63}$ B. Safarzadeh, ${ }^{63, \mathrm{dd}}$ M. Zeinali, ${ }^{63}$ M. Felcini, ${ }^{64}$ M. Grunewald, ${ }^{64}$ M. Abbrescia, ${ }^{65 a, 65 b}$ C. Calabria, ${ }^{65 a, 65 b}$ A. Colaleo, ${ }^{65 a}$ D. Creanza,${ }^{65 a, 65 c}$ L. Cristella ${ }^{65 a, 65 b}$ N. De Filippis ${ }^{65 a, 65 c}$ M. De Palma ${ }^{65 a, 65 b}$ A. Di Florio,${ }^{65 a, 65 b}$ F. Errico,${ }^{65 a, 65 b}$ L. Fiore, ${ }^{65 a}$ A. Gelmi, ${ }^{65 a, 65 b}$ 
G. Iaselli, ${ }^{65 a, 65 c}$ M. Ince, ${ }^{65 a, 65 b}$ S. Lezki, ${ }^{65 a, 65 b}$ G. Maggi, ${ }^{65 a, 65 c}$ M. Maggi, ${ }^{65 a}$ G. Miniello, ${ }^{65 a, 65 b}$ S. My, ${ }^{65 a, 65 b}$ S. Nuzzo, ${ }^{65 a, 65 b}$ A. Pompili, ${ }^{65 a, 65 b}$ G. Pugliese,${ }^{65 a, 65 c}$ R. Radogna ${ }^{65 a}$ A. Ranieri, ${ }^{65 a}$ G. Selvaggi, ${ }^{65 a, 65 b}$ L. Silvestris, ${ }^{65 a}$ R. Venditti, ${ }^{65 a}$ P. Verwilligen, ${ }^{65 a}$ G. Abbiendi, ${ }^{66 a}$ C. Battilana,${ }^{66 a, 66 b}$ D. Bonacorsi, ${ }^{66 a, 66 b}$ L. Borgonovi, ${ }^{66 a, 66 b}$ S. Braibant-Giacomelli, ${ }^{66 a, 66 b}$ R. Campanini, ${ }^{66,66 \mathrm{~b}}$ P. Capiluppi, ${ }^{66,66 \mathrm{~b}}$ A. Castro, ${ }^{66 a, 66 \mathrm{~b}}$ F. R. Cavallo, ${ }^{66 \mathrm{a}}$ S. S. Chhibra, ${ }^{66,66 \mathrm{~b}}$ G. Codispoti, ${ }^{66 a, 66 \mathrm{~b}}$ M. Cuffiani ${ }^{66 a, 66 b}$ G. M. Dallavalle, ${ }^{66 a}$ F. Fabbri, ${ }^{66 a}$ A. Fanfani, ${ }^{66 a, 66 b}$ E. Fontanesi, ${ }^{66 a}$ P. Giacomelli, ${ }^{66 a}$ C. Grandi, ${ }^{66 a}$ L. Guiducci, ${ }^{66 a, 66 b}$ F. Iemmi, ${ }^{66 a, 66 b}$ S. Lo Meo, ${ }^{66 a, e e}$ S. Marcellini, ${ }^{66 a}$ G. Masetti, ${ }^{66 a}$ A. Montanari, ${ }^{66 a}$ F. L. Navarria, ${ }^{66 a, 66 b}$ A. Perrotta, ${ }^{66 \mathrm{a}}$ F. Primavera, ${ }^{66 \mathrm{a}, 66 \mathrm{~b}}$ A. M. Rossi, ${ }^{66 a, 66 \mathrm{~b}}$ T. Rovelli, ${ }^{66 \mathrm{a}, 66 \mathrm{~b}}$ G. P. Siroli,${ }^{66 a, 66 \mathrm{~b}}$ N. Tosi, ${ }^{66 \mathrm{a}}$ S. Albergo, ${ }^{67,67 b, f f}$ A. Di Mattia, ${ }^{67 a}$ R. Potenza, ${ }^{67 a, 67 b}$ A. Tricomi ${ }^{67 a, 67 b, f f}$ C. Tuve, ${ }^{67 a, 67 b}$ G. Barbagli, ${ }^{68 a}$ K. Chatterjee, ${ }^{68 a, 68 b}$ V. Ciulli, ${ }^{68 a, 68 b}$ C. Civinini, ${ }^{68 \mathrm{a}}$ R. D’Alessandro, ${ }^{68 \mathrm{a}, 68 \mathrm{~b}}$ E. Focardi, ${ }^{68 \mathrm{a}, 68 \mathrm{~b}}$ G. Latino, ${ }^{68 \mathrm{a}}$ P. Lenzi, ${ }^{68 \mathrm{a}, 68 \mathrm{~b}}$ M. Meschini, ${ }^{68 \mathrm{a}}$ S. Paoletti, ${ }^{68 \mathrm{a}}$ L. Russo, ${ }^{68 a, g g}$ G. Sguazzoni, ${ }^{68 a}$ D. Strom, ${ }^{68 a}$ L. Viliani, ${ }^{68 a}$ L. Benussi ${ }^{69}$ S. Bianco, ${ }^{69}$ F. Fabbri, ${ }^{69}$ D. Piccolo, ${ }^{69}$ F. Ferro, ${ }^{70 a}$ R. Mulargia, ${ }^{70 a, 70 b}$ E. Robutti, ${ }^{70 a}$ S. Tosi, ${ }^{70 a, 70 b}$ A. Benaglia, ${ }^{71 a}$ A. Beschi, ${ }^{71 a, 71 b}$ F. Brivio, ${ }^{71 a, 71 b}$ V. Ciriolo, ${ }^{71 a, 71 b, r}$ S. Di Guida,${ }^{71 a, 71 b, r}$ M. E. Dinardo, ${ }^{71 a, 71 b}$ S. Fiorendi, ${ }^{71 a, 71 b}$ S. Gennai, ${ }^{71 a}$ A. Ghezzi, ${ }^{71 a, 71 b}$ P. Govoni, ${ }^{71 a, 71 b}$ M. Malberti, ${ }^{71 a, 71 b}$ S. Malvezzi, ${ }^{71 a}$ D. Menasce, ${ }^{71 a}$ F. Monti, ${ }^{71 a}$ L. Moroni, ${ }^{71 a}$ M. Paganoni, ${ }^{71,71 b}$ D. Pedrini, ${ }^{71 a}$ S. Ragazzi, ${ }^{71 a, 71 b}$ T. Tabarelli de Fatis, ${ }^{71 \mathrm{a}, 71 \mathrm{~b}}$ D. Zuolo, ${ }^{71 \mathrm{a}, 71 \mathrm{~b}}$ S. Buontempo, ${ }^{72 \mathrm{a}}$ N. Cavallo, ${ }^{72 \mathrm{a}, 72 \mathrm{c}}$ A. De Iorio, ${ }^{72 \mathrm{a}, 72 \mathrm{~b}}$ A. Di Crescenzo, ${ }^{72 \mathrm{a}, 72 \mathrm{~b}}$ F. Fabozzi, ${ }^{72 a, 72 c}$ F. Fienga, ${ }^{72 a}$ G. Galati, ${ }^{72 a}$ A. O. M. Iorio, ${ }^{72 a, 72 b}$ L. Lista, ${ }^{72 a}$ S. Meola, ${ }^{72 a, 72 d, r}$ P. Paolucci, ${ }^{72 a, r}$ C. Sciacca, ${ }^{72 a, 72 b}$ E. Voevodina, ${ }^{72 a, 72 b}$ P. Azzi,${ }^{73 a}$ N. Bacchetta, ${ }^{73 a}$ D. Bisello, ${ }^{73 a, 73 b}$ A. Boletti, ${ }^{73 a, 73 b}$ A. Bragagnolo, ${ }^{73 a}$ R. Carlin, ${ }^{73 a, 73 b}$ P. Checchia, ${ }^{73 a}$ M. Dall'Osso, ${ }^{73 a, 73 b}$ P. De Castro Manzano, ${ }^{73 a}$ T. Dorigo, ${ }^{73 a}$ U. Dosselli, ${ }^{73 a}$ F. Gasparini, ${ }^{73 a, 73 b}$ U. Gasparini, ${ }^{73 a, 73 b}$ A. Gozzelino, ${ }^{73 a}$ S. Y. Hoh,${ }^{73 a}$ S. Lacaprara ${ }^{73 a}$ P. Lujan, ${ }^{73 a}$ M. Margoni, ${ }^{73 a, 73 b}$ A. T. Meneguzzo, ${ }^{73 a, 73 b}$ J. Pazzini, ${ }^{73 a, 73 b}$ M. Presilla,${ }^{73 a, 73 b}$ P. Ronchese, ${ }^{73 a, 73 b}$ R. Rossin, ${ }^{73 a, 73 b}$ F. Simonetto, ${ }^{73 a, 73 b}$ A. Tiko, ${ }^{73 a}$ E. Torassa ${ }^{73 a}$ M. Tosi ${ }^{73 a, 73 b}$ M. Zanetti, ${ }^{73 a, 73 b}$ P. Zotto, ${ }^{73 a, 73 b}$ G. Zumerle, ${ }^{73 a, 73 b}$ A. Braghieri, ${ }^{74 a}$ A. Magnani, ${ }^{74 a}$ P. Montagna, ${ }^{74 a, 74 b}$ S. P. Ratti, ${ }^{74 a, 74 b}$ V. Re, ${ }^{74 a}$ M. Ressegotti, ${ }^{74,74 b}$ C. Riccardi, ${ }^{74,74 b}$ P. Salvini, ${ }^{74 a}$ I. Vai, ${ }^{74 a, 74 b}$ P. Vitulo, ${ }^{74 a, 74 b}$ M. Biasini, ${ }^{75 a, 75 b}$ G. M. Bilei, ${ }^{75 a}$ C. Cecchi, ${ }^{75 a, 75 b}$ D. Ciangottini, ${ }^{75 a, 75 b}$ L. Fanò, ${ }^{75 a, 75 b}$ P. Lariccia, ${ }^{75 a, 75 b}$ R. Leonardi, ${ }^{75 a, 75 b}$ E. Manoni, ${ }^{75 a}$ G. Mantovani, ${ }^{75 a, 75 b}$ V. Mariani, ${ }^{75 a, 75 b}$ M. Menichelli, ${ }^{75 a}$ A. Rossi, ${ }^{75 a, 75 b}$ A. Santocchia, ${ }^{75 a, 75 b}$ D. Spiga ${ }^{75 a}$ K. Androsov, ${ }^{76 a}$ P. Azzurri, ${ }^{76 a}$ G. Bagliesi, ${ }^{76 a}$ L. Bianchini, ${ }^{76 a}$ T. Boccali, ${ }^{76 a}$ L. Borrello, ${ }^{76 a}$ R. Castaldi, ${ }^{76 a}$ M. A. Ciocci, ${ }^{76 a, 76 b}$ R. Dell'Orso, ${ }^{76 a}$ G. Fedi, ${ }^{76 a}$ F. Fiori, ${ }^{76 a, 76 c}$ L. Giannini, ${ }^{76 a, 76 c}$ A. Giassi, ${ }^{76 a}$ M. T. Grippo, ${ }^{76 a}$ F. Ligabue, ${ }^{76 a, 76 c}$ E. Manca,${ }^{76 a, 76 c}$ G. Mandorli, ${ }^{76 a, 76 c}$ A. Messineo, ${ }^{76 a, 76 b}$ F. Palla, ${ }^{76 a}$ A. Rizzi, ${ }^{76 a, 76 b}$ G. Rolandi ${ }^{76 a, h h}$ A. Scribano, ${ }^{76 a}$ P. Spagnolo ${ }^{76 a}$ R. Tenchini, ${ }^{76 a}$ G. Tonelli, ${ }^{76 a}, 76 \mathrm{~b}$ A. Venturi, ${ }^{76 a}$ P. G. Verdini, ${ }^{76 a}$ L. Barone,${ }^{77 a, 77 b}$ F. Cavallari, ${ }^{77 a}$ M. Cipriani, ${ }^{77 a, 77 b}$ D. Del Re ${ }^{77 a, 77 b}$ E. Di Marco,${ }^{77 a, 77 b}$ M. Diemoz,${ }^{77 a}$ S. Gelli, ${ }^{77,77 b}$ E. Longo,${ }^{77 a, 77 b}$ B. Marzocchi, ${ }^{77 a, 77 b}$ P. Meridiani, ${ }^{77 a}$ G. Organtini, ${ }^{77 a, 77 b}$ F. Pandolfi, ${ }^{77 a}$ R. Paramatti, ${ }^{77,77 b}$ F. Preiato, ${ }^{77,77 b}$ C. Quaranta, ${ }^{77,77 b}$ S. Rahatlou, ${ }^{77 a, 77 b}$ C. Rovelli, ${ }^{77 a}$ F. Santanastasio, ${ }^{77 a, 77 b}$ N. Amapane, ${ }^{78 a, 78 b}$ R. Arcidiacono, ${ }^{78 a, 78 c}$ S. Argiro, ${ }^{78 a, 78 b}$ M. Arneodo, ${ }^{78 a, 78 c}$ N. Bartosik, ${ }^{78 a}$ R. Bellan, ${ }^{78 a, 78 b}$ C. Biino, ${ }^{78 a}$ A. Cappati, ${ }^{78 a, 78 b}$ N. Cartiglia, ${ }^{78 a}$ F. Cenna, ${ }^{78 a, 78 b}$ S. Cometti,${ }^{78 a}$ M. Costa, ${ }^{78 a, 78 b}$

R. Covarelli, ${ }^{78 a, 78 b}$ N. Demaria, ${ }^{78 a}$ B. Kiani, ${ }^{78 a, 78 b}$ C. Mariotti, ${ }^{78 a}$ S. Maselli, ${ }^{78 a}$ E. Migliore, ${ }^{78 a, 78 b}$ V. Monaco, ${ }^{78 a, 78 b}$

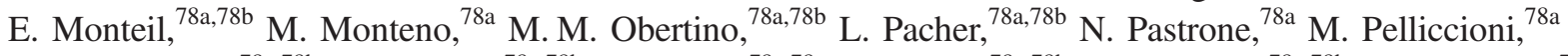

G. L. Pinna Angioni, ${ }^{78,78 b}$ A. Romero, ${ }^{78 a, 78 b}$ M. Ruspa, ${ }^{78 a, 78 c}$ R. Sacchi, ${ }^{78 a, 78 b}$ R. Salvatico, ${ }^{78 a, 78 b}$ K. Shchelina, ${ }^{78 a, 78 b}$ V. Sola, ${ }^{78 a}$ A. Solano, ${ }^{78 a, 78 b}$ D. Soldi, ${ }^{78 a, 78 b}$ A. Staiano, ${ }^{78 a}$ S. Belforte, ${ }^{79 a}$ V. Candelise, ${ }^{79 a, 79 b}$ M. Casarsa, ${ }^{79 a}$ F. Cossutti, ${ }^{79 a}$ A. Da Rold, ${ }^{79 a, 79 b}$ G. Della Ricca ${ }^{79 a, 79 b}$ F. Vazzoler, ${ }^{79 a, 79 b}$ A. Zanetti, ${ }^{79 a}$ D. H. Kim,${ }^{80}$ G. N. Kim, ${ }^{80}$ M. S. Kim,${ }^{80}$ J. Lee, ${ }^{80}$ S. W. Lee,${ }^{80}$ C. S. Moon, ${ }^{80}$ Y. D. Oh, ${ }^{80}$ S. I. Pak ${ }^{80}$ S. Sekmen,${ }^{80}$ D. C. Son, ${ }^{80}$ Y. C. Yang, ${ }^{80}$ H. Kim, ${ }^{81}$ D. H. Moon, ${ }^{81}$ G. Oh,${ }^{81}$ B. Francois, ${ }^{82}$ J. Goh, ${ }^{82, i i}$ T. J. Kim, ${ }^{82}$ S. Cho, ${ }^{83}$ S. Choi, ${ }^{83}$ Y. Go ${ }^{83}$ D. Gyun,${ }^{83}$ S. Ha, ${ }^{83}$ B. Hong ${ }^{83}$ Y. Jo, ${ }^{83}$ K. Lee, ${ }^{83}$ K. S. Lee, ${ }^{83}$ S. Lee, ${ }^{83}$ J. Lim, ${ }^{83}$ S. K. Park, ${ }^{83}$ Y. Roh, ${ }^{83}$ H. S. Kim, ${ }^{84}$ J. Almond ${ }^{85}$ J. Kim,${ }^{85}$ J. S. Kim, ${ }^{85}$ H. Lee, ${ }^{85}$ K. Lee, ${ }^{85}$ S. Lee, ${ }^{85}$ K. Nam, ${ }^{85}$ S. B. Oh, ${ }^{85}$ B. C. Radburn-Smith, ${ }^{85}$ S. h. Seo, ${ }^{85}$ U. K. Yang, ${ }^{85}$ H. D. Yoo, ${ }^{85}$ G. B. Yu, ${ }^{85}$ D. Jeon, ${ }^{86}$ H. Kim, ${ }^{86}$ J. H. Kim, ${ }^{86}$ J. S. H. Lee, ${ }^{86}$ I. C. Park, ${ }^{86}$ Y. Choi, ${ }^{87}$ C. Hwang, ${ }^{87}$ J. Lee, ${ }^{87}$ I. Yu ${ }^{87}$ V. Veckalns, ${ }^{88, j j}$ V. Dudenas, ${ }^{89}$ A. Juodagalvis ${ }^{89}$ J. Vaitkus ${ }^{89}$ Z. A. Ibrahim, ${ }^{90}$ M. A. B. Md Ali, ${ }^{90, k k}$ F. Mohamad Idris, ${ }^{90,11}$ W. A. T. Wan Abdullah, ${ }^{90}$ M. N. Yusli, ${ }^{90}$ Z. Zolkapli, ${ }^{90}$ J. F. Benitez, ${ }^{91}$ A. Castaneda Hernandez, ${ }^{91}$ J. A. Murillo Quijada, ${ }^{91}$ H. Castilla-Valdez, ${ }^{92}$ E. De La Cruz-Burelo, ${ }^{92}$ M. C. Duran-Osuna, ${ }^{92}$ I. Heredia-De La Cruz, ${ }^{92, m m}$ R. Lopez-Fernandez, ${ }^{92}$ J. Mejia Guisao, ${ }^{92}$ R. I. Rabadan-Trejo, ${ }^{92}$ G. Ramirez-Sanchez, ${ }^{92}$ R. Reyes-Almanza, ${ }^{92}$ A. Sanchez-Hernandez, ${ }^{92}$ S. Carrillo Moreno, ${ }^{93}$ C. Oropeza Barrera, ${ }^{93}$ M. Ramirez-Garcia, ${ }^{93}$ F. Vazquez Valencia, ${ }^{93}$ J. Eysermans, ${ }^{94}$ I. Pedraza, ${ }^{94}$ H. A. Salazar Ibarguen, ${ }^{94}$ C. Uribe Estrada ${ }^{94}$ A. Morelos Pineda, ${ }^{95}$ N. Raicevic, ${ }^{96}$ D. Krofcheck, ${ }^{97}$ S. Bheesette, ${ }^{98}$ P. H. Butler, ${ }^{98}$ A. Ahmad, ${ }^{99}$ M. Ahmad, ${ }^{99}$ M. I. Asghar, ${ }^{99}$ Q. Hassan, ${ }^{99}$ H. R. Hoorani, ${ }^{99}$ W. A. Khan, ${ }^{99}$ M. A. Shah,${ }^{99}$ M. Shoaib, ${ }^{99}$ M. Waqas, ${ }^{99}$ H. Bialkowska, ${ }^{100}$ M. Bluj, ${ }^{100}$ B. Boimska, ${ }^{100}$ T. Frueboes, ${ }^{100}$ M. Górski, ${ }^{100}$ M. Kazana, ${ }^{100}$ M. Szleper, ${ }^{100}$ P. Traczyk, ${ }^{100}$ 
P. Zalewski, ${ }^{100}$ K. Bunkowski, ${ }^{101}$ A. Byszuk, ${ }^{101, n n}$ K. Doroba, ${ }^{101}$ A. Kalinowski, ${ }^{101}$ M. Konecki, ${ }^{101}$ J. Krolikowski, ${ }^{101}$ M. Misiura, ${ }^{101}$ M. Olszewski, ${ }^{101}$ A. Pyskir, ${ }^{101}$ M. Walczak, ${ }^{101}$ M. Araujo, ${ }^{102}$ P. Bargassa, ${ }^{102}$ C. Beirão Da Cruz E Silva, ${ }^{102}$ A. Di Francesco, ${ }^{102}$ P. Faccioli, ${ }^{102}$ B. Galinhas, ${ }^{102}$ M. Gallinaro, ${ }^{102}$ J. Hollar, ${ }^{102}$ N. Leonardo, ${ }^{102}$ J. Seixas, ${ }^{102}$ G. Strong, ${ }^{102}$ O. Toldaiev, ${ }^{102}$ J. Varela, ${ }^{102}$ S. Afanasiev, ${ }^{103}$ P. Bunin, ${ }^{103}$ M. Gavrilenko, ${ }^{103}$ I. Golutvin, ${ }^{103}$ I. Gorbunov, ${ }^{103}$ A. Kamenev, ${ }^{103}$ V. Karjavine, ${ }^{103}$ A. Lanev, ${ }^{103}$ A. Malakhov, ${ }^{103}$ V. Matveev, ${ }^{103, o o, p p}$ P. Moisenz, ${ }^{103}$ V. Palichik, ${ }^{103}$ V. Perelygin,,${ }^{103}$ S. Shmatov, ${ }^{103}$ S. Shulha, ${ }^{103}$ N. Skatchkov, ${ }^{103}$ V. Smirnov, ${ }^{103}$ N. Voytishin, ${ }^{103}$ A. Zarubin, ${ }^{103}$ V. Golovtsov, ${ }^{104}$ Y. Ivanov, ${ }^{104}$ V. Kim, ${ }^{104, q q}$ E. Kuznetsova, ${ }^{104, \text { rr }}$ P. Levchenko, ${ }^{104}$ V. Murzin, ${ }^{104}$ V. Oreshkin,,${ }^{104}$ I. Smirnov, ${ }^{104}$ D. Sosnov, ${ }^{104}$ V. Sulimov, ${ }^{104}$ L. Uvarov, ${ }^{104}$ S. Vavilov, ${ }^{104}$ A. Vorobyev, ${ }^{104}$ Yu. Andreev, ${ }^{105}$ A. Dermenev, ${ }^{105}$ S. Gninenko, ${ }^{105}$ N. Golubev, ${ }^{105}$ A. Karneyeu, ${ }^{105}$ M. Kirsanov, ${ }^{105}$ N. Krasnikov, ${ }^{105}$ A. Pashenkov, ${ }^{105}$ A. Shabanov, ${ }^{105}$ D. Tlisov, ${ }^{105}$ A. Toropin, ${ }^{105}$ V. Epshteyn, ${ }^{106}$ V. Gavrilov, ${ }^{106}$ N. Lychkovskaya, ${ }^{106}$ V. Popov, ${ }^{106}$ I. Pozdnyakov, ${ }^{106}$ G. Safronov, ${ }^{106}$ A. Spiridonov, ${ }^{106}$ A. Stepennov, ${ }^{106}$ V. Stolin, ${ }^{106}$ M. Toms, ${ }^{106}$ E. Vlasov, ${ }^{106}$ A. Zhokin, ${ }^{106}$ T. Aushev, ${ }^{107}$ R. Chistov, ${ }^{108, s s}$ M. Danilov, ${ }^{108, s s}$ S. Polikarpov, ${ }^{108, s s}$ E. Tarkovskii, ${ }^{108}$ V. Andreev ${ }^{109}$ M. Azarkin, ${ }^{109}$ I. Dremin, ${ }^{109, p p}$ M. Kirakosyan, ${ }^{109}$ A. Terkulov, ${ }^{109}$ A. Belyaev, ${ }^{110}$ E. Boos, ${ }^{110}$ M. Dubinin, ${ }^{110, t \mathrm{t}}$ L. Dudko, ${ }^{110}$ A. Ershov, ${ }^{110}$ A. Gribushin, ${ }^{110}$ V. Klyukhin, ${ }^{110}$ O. Kodolova, ${ }^{110}$ I. Lokhtin, ${ }^{110}$ S. Obraztsov, ${ }^{110}$ S. Petrushanko, ${ }^{110}$ V. Savrin, ${ }^{110}$ A. Snigirev, ${ }^{110}$ A. Barnyakov, ${ }^{111, u u}$ V. Blinov, ${ }^{111, \text { uu }}$ T. Dimova, ${ }^{111 \text {,u }}$ L. Kardapoltsev, ${ }^{111 \text {,u }}$ Y. Skovpen, ${ }^{11, \text { uu }}$ I. Azhgirey, ${ }^{112}$ I. Bayshev, ${ }^{112}$ S. Bitioukov, ${ }^{112}$ V. Kachanov, ${ }^{112}$ A. Kalinin, ${ }^{112}$ D. Konstantinov, ${ }^{112}$ P. Mandrik, ${ }^{112}$ V. Petrov,${ }^{112}$ R. Ryutin, ${ }^{112}$ S. Slabospitskii, ${ }^{112}$ A. Sobol,${ }^{112}$ S. Troshin, ${ }^{112}$ N. Tyurin, ${ }^{112}$ A. Uzunian, ${ }^{112}$ A. Volkov, ${ }^{112}$ A. Babaev, ${ }^{113}$ S. Baidali, ${ }^{113}$ A. Iuzhakov, ${ }^{113}$ V. Okhotnikov, ${ }^{113}$ P. Adzic, ${ }^{114, v v}$ P. Cirkovic, ${ }^{114}$ D. Devetak, ${ }^{114}$ M. Dordevic, ${ }^{114}$ P. Milenovic, ${ }^{114, w w}$ J. Milosevic, ${ }^{114}$ J. Alcaraz Maestre, ${ }^{115}$

A. Álvarez Fernández, ${ }^{115}$ I. Bachiller, ${ }^{115}$ M. Barrio Luna, ${ }^{115}$ J. A. Brochero Cifuentes, ${ }^{115}$ M. Cerrada, ${ }^{115}$ N. Colino, ${ }^{115}$ B. De La Cruz, ${ }^{115}$ A. Delgado Peris, ${ }^{115}$ C. Fernandez Bedoya, ${ }^{115}$ J. P. Fernández Ramos, ${ }^{115}$ J. Flix, ${ }^{115}$ M. C. Fouz, ${ }^{115}$ O. Gonzalez Lopez, ${ }^{115}$ S. Goy Lopez, ${ }^{115}$ J. M. Hernandez, ${ }^{115}$ M. I. Josa ${ }^{115}$ D. Moran, ${ }^{115}$ A. Pérez-Calero Yzquierdo, ${ }^{115}$ J. Puerta Pelayo, ${ }^{115}$ I. Redondo, ${ }^{115}$ L. Romero, ${ }^{115}$ S. Sánchez Navas, ${ }^{115}$ M. S. Soares, ${ }^{115}$ A. Triossi, ${ }^{115}$ C. Albajar, ${ }^{16}$ J. F. de Trocóniz, ${ }^{116}$ J. Cuevas, ${ }^{117}$ C. Erice ${ }^{117}$ J. Fernandez Menendez, ${ }^{117}$ S. Folgueras, ${ }^{117}$ I. Gonzalez Caballero, ${ }^{117}$ J. R. González Fernández, ${ }^{117}$ E. Palencia Cortezon, ${ }^{117}$ V. Rodríguez Bouza,${ }^{117}$ S. Sanchez Cruz,${ }^{117}$ J. M. Vizan Garcia, ${ }^{117}$ I. J. Cabrillo, ${ }^{118}$ A. Calderon, ${ }^{118}$ B. Chazin Quero, ${ }^{118}$ J. Duarte Campderros, ${ }^{118}$ M. Fernandez, ${ }^{118}$ P. J. Fernández Manteca, ${ }^{118}$ A. García Alonso, ${ }^{118}$ G. Gomez, ${ }^{118}$ A. Lopez Virto, ${ }^{118}$ C. Martinez Rivero, ${ }^{118}$ P. Martinez Ruiz del Arbol, ${ }^{118}$ F. Matorras, ${ }^{118}$ J. Piedra Gomez, ${ }^{118}$ C. Prieels, ${ }^{118}$ T. Rodrigo, ${ }^{118}$ A. Ruiz-Jimeno, ${ }^{118}$ L. Scodellaro, ${ }^{118}$ N. Trevisani, ${ }^{118}$ I. Vila, ${ }^{118}$ N. Wickramage, ${ }^{119}$ D. Abbaneo, ${ }^{120}$ B. Akgun, ${ }^{120}$ E. Auffray, ${ }^{120}$ G. Auzinger, ${ }^{120}$ P. Baillon, ${ }^{120, a}$ A. H. Ball, ${ }^{120}$ D. Barney, ${ }^{120}$ J. Bendavid, ${ }^{120}$ M. Bianco, ${ }^{120}$ A. Bocci, ${ }^{120}$ C. Botta, ${ }^{120}$ E. Brondolin, ${ }^{120}$ T. Camporesi, ${ }^{120}$ M. Cepeda, ${ }^{120}$ G. Cerminara, ${ }^{120}$ E. Chapon, ${ }^{120}$ Y. Chen, ${ }^{120}$ G. Cucciati, ${ }^{120}$ D. d'Enterria, ${ }^{120}$ A. Dabrowski, ${ }^{120}$ N. Daci, ${ }^{120}$ V. Daponte, ${ }^{120}$ A. David, ${ }^{120}$ A. De Roeck, ${ }^{120}$ N. Deelen, ${ }^{120}$ M. Dobson, ${ }^{120}$ M. Dünser, ${ }^{120}$ N. Dupont,${ }^{120}$ A. Elliott-Peisert, ${ }^{120}$ F. Fallavollita, ${ }^{120, x x}$ D. Fasanella, ${ }^{120}$ G. Franzoni, ${ }^{120}$ J. Fulcher, ${ }^{120}$ W. Funk, ${ }^{120}$ D. Gigi, ${ }^{120}$ A. Gilbert, ${ }^{120}$ K. Gill, ${ }^{120}$ F. Glege, ${ }^{120}$ M. Gruchala, ${ }^{120}$ M. Guilbaud, ${ }^{120}$ D. Gulhan, ${ }^{120}$ J. Hegeman, ${ }^{120}$ C. Heidegger, ${ }^{120}$ Y. Iiyama, ${ }^{120}$ V. Innocente, ${ }^{120}$ G. M. Innocenti, ${ }^{120}$ A. Jafari, ${ }^{120}$ P. Janot, ${ }^{120}$ O. Karacheban, ${ }^{120, \mathrm{u}}$ J. Kieseler, ${ }^{120}$ A. Kornmayer, ${ }^{120}$ M. Krammer, ${ }^{120, b}$ C. Lange, ${ }^{120}$ P. Lecoq, ${ }^{120}$ C. Lourenço, ${ }^{120}$ L. Malgeri, ${ }^{120}$ M. Mannelli, ${ }^{120}$ A. Massironi, ${ }^{120}$ F. Meijers, ${ }^{120}$ J. A. Merlin, ${ }^{120}$ S. Mersi, ${ }^{120}$ E. Meschi, ${ }^{120}$ F. Moortgat ${ }^{120}$ M. Mulders,${ }^{120}$ J. Ngadiuba, ${ }^{120}$ S. Nourbakhsh, ${ }^{120}$ S. Orfanelli, ${ }^{120}$ L. Orsini, ${ }^{120}$ F. Pantaleo, ${ }^{120, r}$ L. Pape,${ }^{120}$ E. Perez, ${ }^{120}$ M. Peruzzi, ${ }^{120}$ A. Petrilli, ${ }^{120}$ G. Petrucciani ${ }^{120}$ A. Pfeiffer,${ }^{120}$ M. Pierini, ${ }^{120}$ F. M. Pitters, ${ }^{120}$ D. Rabady,${ }^{120}$ A. Racz, ${ }^{120}$ M. Rovere ${ }^{120}$ H. Sakulin, ${ }^{120}$ C. Schäfer, ${ }^{120}$ C. Schwick, ${ }^{120}$ M. Selvaggi, ${ }^{120}$ A. Sharma, ${ }^{120}$ P. Silva, ${ }^{120}$ P. Sphicas, ${ }^{120, y y}$ A. Stakia, ${ }^{120}$ J. Steggemann, ${ }^{120}$ V. R. Tavolaro, ${ }^{120}$ D. Treille, ${ }^{120}$ A. Tsirou, ${ }^{120}$ A. Vartak,${ }^{120}$ M. Verzetti, ${ }^{120}$ W. D. Zeuner, ${ }^{120}$ L. Caminada, ${ }^{121, z z}$ K. Deiters, ${ }^{121}$ W. Erdmann, ${ }^{121}$ R. Horisberger ${ }^{121}$ Q. Ingram, ${ }^{121}$ H. C. Kaestli, ${ }^{121}$ D. Kotlinski, ${ }^{121}$ U. Langenegger, ${ }^{121}$ T. Rohe, ${ }^{121}$ S. A. Wiederkehr, ${ }^{121}$ M. Backhaus, ${ }^{122}$ P. Berger, ${ }^{122}$ N. Chernyavskaya, ${ }^{122}$ G. Dissertori, ${ }^{122}$ M. Dittmar, ${ }^{122}$ M. Donegà, ${ }^{122}$ C. Dorfer, ${ }^{122}$ T. A. Gómez Espinosa, ${ }^{122}$ C. Grab, ${ }^{122}$ D. Hits, ${ }^{122}$ T. Klijnsma, ${ }^{122}$ W. Lustermann, ${ }^{122}$ R. A. Manzoni, ${ }^{122}$ M. Marionneau, ${ }^{122}$ M. T. Meinhard,${ }^{122}$ F. Micheli, ${ }^{122}$ P. Musella, ${ }^{122}$

F. Nessi-Tedaldi, ${ }^{122}$ F. Pauss ${ }^{122}$ G. Perrin, ${ }^{122}$ L. Perrozzi, ${ }^{122}$ S. Pigazzini,${ }^{122}$ M. Reichmann,${ }^{122}$ C. Reissel, ${ }^{122}$ T. Reitenspiess, ${ }^{122}$ D. Ruini, ${ }^{122}$ D. A. Sanz Becerra, ${ }^{122}$ M. Schönenberger, ${ }^{122}$ L. Shchutska ${ }^{122}$ K. Theofilatos, ${ }^{122}$ M. L. Vesterbacka Olsson, ${ }^{122}$ R. Wallny, ${ }^{122}$ D. H. Zhu, ${ }^{122}$ T. K. Aarrestad, ${ }^{123}$ C. Amsler, ${ }^{123, a a a}$ D. Brzhechko, ${ }^{123}$ M. F. Canelli, ${ }^{123}$ A. De Cosa, ${ }^{123}$ R. Del Burgo, ${ }^{123}$ S. Donato, ${ }^{123}$ C. Galloni, ${ }^{123}$ T. Hreus, ${ }^{123}$ B. Kilminster, ${ }^{123}$ S. Leontsinis, ${ }^{123}$ V. M. Mikuni, ${ }^{123}$ I. Neutelings, ${ }^{123}$ G. Rauco, ${ }^{123}$ P. Robmann, ${ }^{123}$ D. Salerno, ${ }^{123}$ K. Schweiger, ${ }^{123}$ C. Seitz, ${ }^{123}$ Y. Takahashi, ${ }^{123}$ S. Wertz, ${ }^{123}$ A. Zucchetta, ${ }^{123}$ T. H. Doan, ${ }^{124}$ C. M. Kuo, ${ }^{124}$ W. Lin, ${ }^{124}$ S. S. Yu, ${ }^{124}$ P. Chang, ${ }^{125}$ Y. Chao, ${ }^{125}$ K. F. Chen, ${ }^{125}$ 
P. H. Chen, ${ }^{125}$ W.-S. Hou, ${ }^{125}$ Y. F. Liu, ${ }^{125}$ R.-S. Lu, ${ }^{125}$ E. Paganis, ${ }^{125}$ A. Psallidas, ${ }^{125}$ A. Steen, ${ }^{125}$ B. Asavapibhop, ${ }^{126}$ N. Srimanobhas, ${ }^{126}$ N. Suwonjandee, ${ }^{126}$ A. Bat ${ }^{127}$ F. Boran, ${ }^{127}$ S. Cerci, ${ }^{127, b b b}$ S. Damarseckin, ${ }^{127, c c c}$ Z. S. Demiroglu, ${ }^{127}$ F. Dolek, ${ }^{127}$ C. Dozen, ${ }^{127}$ I. Dumanoglu, ${ }^{127}$ G. Gokbulut, ${ }^{127}$ Emine Gurpinar Guler, ${ }^{127, \text { ddd }}$ Y. Guler, ${ }^{127}$ I. Hos, ${ }^{127, \text { eee }}$ C. Isik, ${ }^{127}$ E. E. Kangal ${ }^{127, f f f}$ O. Kara, ${ }^{127}$ A. Kayis Topaksu, ${ }^{127}$ U. Kiminsu, ${ }^{127}$ M. Oglakci, ${ }^{127}$ G. Onengut, ${ }^{127}$ K. Ozdemir, ${ }^{127, g g g}$ S. Ozturk, ${ }^{127, \text { hhh }}$ D. Sunar Cerci, ${ }^{127, b b b}$ B. Tali, ${ }^{127, b b b}$ U. G. Tok, ${ }^{127}$ S. Turkcapar, ${ }^{127}$ I. S. Zorbakir, ${ }^{127}$ C. Zorbilmez, ${ }^{127}$ B. Isildak, ${ }^{128, i i i}$ G. Karapinar, ${ }^{128, j j i}$ M. Yalvac, ${ }^{128}$ M. Zeyrek, ${ }^{128}$ I. O. Atakisi, ${ }^{129}$ E. Gülmez, ${ }^{129}$ M. Kaya, ${ }^{129, k k k}$ O. Kaya, ${ }^{129,111}$ Ö. Özçelik, ${ }^{129}$ S. Ozkorucuklu, ${ }^{129, m m m}$ S. Tekten, ${ }^{129}$ E. A. Yetkin, ${ }^{129, n n n}$ A. Cakir, ${ }^{130}$ K. Cankocak, ${ }^{130}$ Y. Komurcu, ${ }^{130}$ S. Sen, ${ }^{130,000}$ B. Grynyov, ${ }^{131}$ L. Levchuk, ${ }^{132}$ F. Ball, ${ }^{133}$ J. J. Brooke, ${ }^{133}$ D. Burns,${ }^{133}$ E. Clement, ${ }^{133}$ D. Cussans, ${ }^{133}$ O. Davignon, ${ }^{133}$ H. Flacher, ${ }^{133}$ J. Goldstein, ${ }^{133}$ G. P. Heath ${ }^{133}$ H. F. Heath,${ }^{133}$ L. Kreczko, ${ }^{133}$ D. M. Newbold, ${ }^{133, p p p}$ S. Paramesvaran, ${ }^{133}$ B. Penning, ${ }^{133}$ T. Sakuma, ${ }^{133}$ D. Smith, ${ }^{133}$ V. J. Smith, ${ }^{133}$ J. Taylor, ${ }^{133}$ A. Titterton, ${ }^{133}$ K. W. Bell, ${ }^{134}$ A. Belyaev, ${ }^{134, q q 9}$ C. Brew, ${ }^{134}$ R. M. Brown, ${ }^{134}$ D. Cieri, ${ }^{134}$ D. J. A. Cockerill, ${ }^{134}$ J. A. Coughlan, ${ }^{134}$ K. Harder, ${ }^{134}$ S. Harper, ${ }^{134}$ J. Linacre, ${ }^{134}$ K. Manolopoulos, ${ }^{134}$ E. Olaiya,${ }^{134}$ D. Petyt, ${ }^{134}$ T. Reis, ${ }^{134}$ T. Schuh, ${ }^{134}$ C. H. Shepherd-Themistocleous, ${ }^{134}$ A. Thea, ${ }^{134}$ I. R. Tomalin, ${ }^{134}$ T. Williams, ${ }^{134}$ W. J. Womersley, ${ }^{134}$ R. Bainbridge, ${ }^{135}$ P. Bloch, ${ }^{135}$ J. Borg, ${ }^{135}$ S. Breeze, ${ }^{135}$ O. Buchmuller, ${ }^{135}$ A. Bundock,${ }^{135}$ Gurpreet Singh Chahal, ${ }^{135}$ D. Colling, ${ }^{135}$ P. Dauncey, ${ }^{135}$ G. Davies, ${ }^{135}$ M. Della Negra, ${ }^{135}$ R. Di Maria, ${ }^{135}$ P. Everaerts, ${ }^{135}$ G. Hall, ${ }^{135}$ G. Iles, ${ }^{135}$ T. James, ${ }^{135}$ M. Komm, ${ }^{135}$ C. Laner, ${ }^{135}$ L. Lyons, ${ }^{135}$ A.-M. Magnan, ${ }^{135}$ S. Malik, ${ }^{135}$ A. Martelli, ${ }^{135}$ V. Milosevic, ${ }^{135}$ J. Nash, ${ }^{135, r r r}$ A. Nikitenko, ${ }^{135, \mathrm{i}}$ V. Palladino, ${ }^{135}$ M. Pesaresi, ${ }^{135}$ D. M. Raymond, ${ }^{135}$ A. Richards,${ }^{135}$ A. Rose, ${ }^{135}$ E. Scott, ${ }^{135}$ C. Seez, ${ }^{135}$ A. Shtipliyski, ${ }^{135}$ M. Stoye, ${ }^{135}$ T. Strebler, ${ }^{135}$ S. Summers ${ }^{135}$ A. Tapper,${ }^{135}$ K. Uchida,${ }^{135}$ T. Virdee,${ }^{135, \mathrm{r}}$ N. Wardle, ${ }^{135}$ D. Winterbottom, ${ }^{135}$ J. Wright, ${ }^{135}$ S. C. Zenz, ${ }^{135}$ J. E. Cole, ${ }^{136}$ P. R. Hobson, ${ }^{136}$ A. Khan ${ }^{136}$ P. Kyberd, ${ }^{136}$ C. K. Mackay, ${ }^{136}$ A. Morton, ${ }^{136}$ I. D. Reid,${ }^{136}$ L. Teodorescu, ${ }^{136}$ S. Zahid,${ }^{136}$ K. Call, ${ }^{137}$ J. Dittmann, ${ }^{137}$ K. Hatakeyama,${ }^{137}$ H. Liu, ${ }^{137}$ C. Madrid ${ }^{137}$ B. McMaster, ${ }^{137}$ N. Pastika, ${ }^{137}$ C. Smith,${ }^{137}$ R. Bartek, ${ }^{138}$ A. Dominguez, ${ }^{138}$ A. Buccilli, ${ }^{139}$ O. Charaf,${ }^{139}$ S. I. Cooper, ${ }^{139}$ C. Henderson, ${ }^{139}$ P. Rumerio, ${ }^{139}$ C. West, ${ }^{139}$ D. Arcaro, ${ }^{140}$ T. Bose, ${ }^{140}$ Z. Demiragli, ${ }^{140}$ D. Gastler, ${ }^{140}$ S. Girgis, ${ }^{140}$ D. Pinna, ${ }^{140}$ C. Richardson, ${ }^{140}$ J. Rohlf, ${ }^{140}$ D. Sperka, ${ }^{140}$ I. Suarez, ${ }^{140}$ L. Sulak, ${ }^{140}$ D. Zou, ${ }^{140}$ G. Benelli, ${ }^{141}$ B. Burkle ${ }^{141}$ X. Coubez, ${ }^{141}$ D. Cutts, ${ }^{141}$ M. Hadley, ${ }^{141}$ J. Hakala ${ }^{141}$ U. Heintz, ${ }^{141}$ J. M. Hogan, ${ }^{141, \text { sss }}$ K. H. M. Kwok, ${ }^{141}$ E. Laird, ${ }^{141}$ G. Landsberg, ${ }^{141}$ J. Lee, ${ }^{141}$ Z. Mao, ${ }^{141}$ M. Narain, ${ }^{141}$ S. Sagir, ${ }^{141, t t t}$ R. Syarif,${ }^{141}$ E. Usai, ${ }^{141}$ D. Yu, ${ }^{141}$ R. Band, ${ }^{142}$ C. Brainerd, ${ }^{142}$ R. Breedon, ${ }^{142}$ D. Burns, ${ }^{142}$ M. Calderon De La Barca Sanchez, ${ }^{142}$ M. Chertok, ${ }^{142}$ J. Conway, ${ }^{142}$ R. Conway, ${ }^{142}$ P. T. Cox,${ }^{142}$ R. Erbacher, ${ }^{142}$ C. Flores, ${ }^{142}$ G. Funk, ${ }^{142}$ W. Ko, ${ }^{142}$ O. Kukral, ${ }^{142}$ R. Lander, ${ }^{142}$ M. Mulhearn, ${ }^{142}$ D. Pellett, ${ }^{142}$ J. Pilot, ${ }^{142}$ M. Shi, ${ }^{142}$ D. Stolp,${ }^{142}$ D. Taylor, ${ }^{142}$ K. Tos, ${ }^{142}$ M. Tripathi, ${ }^{142}$ Z. Wang,${ }^{142}$ F. Zhang, ${ }^{142}$ M. Bachtis, ${ }^{143}$ C. Bravo, ${ }^{143}$ R. Cousins, ${ }^{143}$ A. Dasgupta, ${ }^{143}$ A. Florent, ${ }^{143}$ J. Hauser, ${ }^{143}$ M. Ignatenko, ${ }^{143}$ N. Mccoll, ${ }^{143}$ S. Regnard, ${ }^{143}$ D. Saltzberg, ${ }^{143}$ C. Schnaible, ${ }^{143}$ V. Valuev, ${ }^{143}$ E. Bouvier, ${ }^{144}$ K. Burt, ${ }^{144}$ R. Clare,${ }^{144}$ J. W. Gary, ${ }^{144}$ S. M. A. Ghiasi Shirazi, ${ }^{144}$ G. Hanson, ${ }^{144}$ G. Karapostoli, ${ }^{144}$ E. Kennedy, ${ }^{144}$ O. R. Long, ${ }^{144}$ M. Olmedo Negrete, ${ }^{144}$ M. I. Paneva, ${ }^{144}$ W. Si, ${ }^{144}$ L. Wang, ${ }^{144}$ H. Wei, ${ }^{144}$ S. Wimpenny, ${ }^{144}$ B. R. Yates, ${ }^{144}$ J. G. Branson, ${ }^{145}$ P. Chang, ${ }^{145}$ S. Cittolin, ${ }^{145}$ M. Derdzinski, ${ }^{145}$ R. Gerosa, ${ }^{145}$ D. Gilbert, ${ }^{145}$ B. Hashemi, ${ }^{145}$ A. Holzner, ${ }^{145}$ D. Klein, ${ }^{145}$ G. Kole, ${ }^{145}$ V. Krutelyov, ${ }^{145}$ J. Letts, ${ }^{145}$ M. Masciovecchio, ${ }^{145}$ S. May, ${ }^{145}$ D. Olivito, ${ }^{145}$ S. Padhi, ${ }^{145}$ M. Pieri, ${ }^{145}$ V. Sharma, ${ }^{145}$ M. Tadel,${ }^{145}$ J. Wood, ${ }^{145}$ F. Würthwein, ${ }^{145}$ A. Yagil, ${ }^{145}$ G. Zevi Della Porta, ${ }^{145}$ N. Amin, ${ }^{146}$ R. Bhandari, ${ }^{146}$ C. Campagnari, ${ }^{146}$ M. Citron, ${ }^{146}$ V. Dutta, ${ }^{146}$ M. Franco Sevilla, ${ }^{146}$ L. Gouskos, ${ }^{146}$ R. Heller, ${ }^{146}$ J. Incandela, ${ }^{146}$ H. Mei,${ }^{146}$ A. Ovcharova, ${ }^{146}$ H. Qu, ${ }^{146}$ J. Richman, ${ }^{146}$ D. Stuart, ${ }^{146}$ S. Wang, ${ }^{146}$ J. Yoo, ${ }^{146}$ D. Anderson, ${ }^{147}$ A. Bornheim, ${ }^{147}$ J. M. Lawhorn, ${ }^{147}$ N. Lu, ${ }^{147}$ H. B. Newman, ${ }^{147}$ T. Q. Nguyen, ${ }^{147}$ J. Pata, ${ }^{147}$ M. Spiropulu, ${ }^{147}$ J. R. Vlimant, ${ }^{147}$ R. Wilkinson, ${ }^{147}$ S. Xie, ${ }^{147}$ Z. Zhang, ${ }^{147}$ R. Y. Zhu, ${ }^{147}$ M. B. Andrews, ${ }^{148}$ T. Ferguson, ${ }^{148}$ T. Mudholkar, ${ }^{148}$ M. Paulini, ${ }^{148}$ M. Sun, ${ }^{148}$ I. Vorobiev, ${ }^{148}$ M. Weinberg, ${ }^{148}$ J. P. Cumalat, ${ }^{149}$ W. T. Ford, ${ }^{149}$ F. Jensen, ${ }^{149}$ A. Johnson, ${ }^{149}$ E. MacDonald, ${ }^{149}$ T. Mulholland, ${ }^{149}$ R. Patel, ${ }^{149}$ A. Perloff, ${ }^{149}$ K. Stenson, ${ }^{149}$ K. A. Ulmer, ${ }^{149}$ S. R. Wagner, ${ }^{149}$ J. Alexander ${ }^{150}$ J. Chaves, ${ }^{150}$ Y. Cheng, ${ }^{150}$ J. Chu, ${ }^{150}$ A. Datta, ${ }^{150}$ K. Mcdermott, ${ }^{150}$ N. Mirman, ${ }^{150}$ J. Monroy, ${ }^{150}$ J. R. Patterson, ${ }^{150}$ D. Quach, ${ }^{150}$ A. Rinkevicius, ${ }^{150}$ A. Ryd,${ }^{150}$ L. Skinnari, ${ }^{150}$ L. Soffi, ${ }^{150}$ S. M. Tan, ${ }^{150}$ Z. Tao, ${ }^{150}$ J. Thom ${ }^{150}$ J. Tucker ${ }^{150}$ P. Wittich, ${ }^{150}$ M. Zientek, ${ }^{150}$ S. Abdullin, ${ }^{151}$ M. Albrow ${ }^{151}$ M. Alyari, ${ }^{151}$ G. Apollinari, ${ }^{151}$ A. Apresyan, ${ }^{151}$ A. Apyan, ${ }^{151}$ S. Banerjee, ${ }^{151}$ L. A. T. Bauerdick, ${ }^{151}$ A. Beretvas, ${ }^{151}$ J. Berryhill, ${ }^{151}$ P. C. Bhat, ${ }^{151}$ K. Burkett, ${ }^{151}$ J. N. Butler ${ }^{151}$ A. Canepa,${ }^{151}$ G. B. Cerati, ${ }^{151}$ H. W. K. Cheung, ${ }^{151}$ F. Chlebana, ${ }^{151}$ M. Cremonesi, ${ }^{151}$ J. Duarte, ${ }^{151}$ V. D. Elvira, ${ }^{151}$ J. Freeman, ${ }^{151}$ Z. Gecse ${ }^{151}$ E. Gottschalk, ${ }^{151}$ L. Gray, ${ }^{151}$ D. Green, ${ }^{151}$ S. Grünendahl, ${ }^{151}$ O. Gutsche, ${ }^{151}$ J. Hanlon, ${ }^{151}$ R. M. Harris, ${ }^{151}$ S. Hasegawa, ${ }^{151}$ J. Hirschauer, ${ }^{151}$ Z. Hu, ${ }^{151}$ B. Jayatilaka, ${ }^{151}$ S. Jindariani, ${ }^{151}$ M. Johnson, ${ }^{151}$ U. Joshi, ${ }^{151}$ B. Klima, ${ }^{151}$ M. J. Kortelainen, ${ }^{151}$ B. Kreis, ${ }^{151}$ S. Lammel, ${ }^{151}$ D. Lincoln, ${ }^{151}$ R. Lipton, ${ }^{151}$ M. Liu, ${ }^{151}$ T. Liu, ${ }^{151}$ J. Lykken, ${ }^{151}$ 
K. Maeshima, ${ }^{151}$ J. M. Marraffino, ${ }^{151}$ D. Mason, ${ }^{151}$ P. McBride, ${ }^{151}$ P. Merkel,${ }^{151}$ S. Mrenna ${ }^{151}$ S. Nahn, ${ }^{151}$ V. O’Dell, ${ }^{151}$ K. Pedro, ${ }^{151}$ C. Pena,${ }^{151}$ O. Prokofyev, ${ }^{151}$ G. Rakness, ${ }^{151}$ F. Ravera, ${ }^{151}$ A. Reinsvold,${ }^{151}$ L. Ristori, ${ }^{151}$ B. Schneider,${ }^{151}$ E. Sexton-Kennedy, ${ }^{151}$ A. Soha, ${ }^{151}$ W. J. Spalding, ${ }^{151}$ L. Spiegel, ${ }^{151}$ S. Stoynev ${ }^{151}$ J. Strait, ${ }^{151}$ N. Strobbe,${ }^{151}$ L. Taylor, ${ }^{151}$ S. Tkaczyk,${ }^{151}$ N. V. Tran, ${ }^{151}$ L. Uplegger,${ }^{151}$ E. W. Vaandering, ${ }^{151}$ C. Vernieri, ${ }^{151}$ M. Verzocchi, ${ }^{151}$ R. Vidal,${ }^{151}$ M. Wang, ${ }^{151}$ H. A. Weber, ${ }^{151}$ D. Acosta, ${ }^{152}$ P. Avery, ${ }^{152}$ P. Bortignon, ${ }^{152}$ D. Bourilkov, ${ }^{152}$ A. Brinkerhoff, ${ }^{152}$ L. Cadamuro, ${ }^{152}$

A. Carnes, ${ }^{152}$ D. Curry ${ }^{152}$ R. D. Field, ${ }^{152}$ S. V. Gleyzer ${ }^{152}$ B. M. Joshi ${ }^{152}$ J. Konigsberg ${ }^{152}$ A. Korytov ${ }^{152}$ K. H. Lo, ${ }^{152}$ P. Ma ${ }^{152}$ K. Matchev ${ }^{152}$ N. Menendez,${ }^{152}$ G. Mitselmakher,${ }^{152}$ D. Rosenzweig, ${ }^{152}$ K. Shi, ${ }^{152}$ J. Wang, ${ }^{152}$ S. Wang, ${ }^{152}$ X. Zuo, ${ }^{152}$ Y. R. Joshi, ${ }^{153}$ S. Linn, ${ }^{153}$ T. Adams, ${ }^{154}$ A. Askew, ${ }^{154}$ S. Hagopian, ${ }^{154}$ V. Hagopian, ${ }^{154}$ K. F. Johnson, ${ }^{154}$ R. Khurana, ${ }^{154}$ T. Kolberg, ${ }^{154}$ G. Martinez, ${ }^{154}$ T. Perry, ${ }^{154}$ H. Prosper, ${ }^{154}$ A. Saha, ${ }^{154}$ C. Schiber, ${ }^{154}$ R. Yohay, ${ }^{154}$ M. M. Baarmand, ${ }^{155}$ V. Bhopatkar, ${ }^{155}$ S. Colafranceschi, ${ }^{155}$ M. Hohlmann,${ }^{155}$ D. Noonan, ${ }^{155}$ M. Rahmani, ${ }^{155}$ T. Roy, ${ }^{155}$ M. Saunders ${ }^{155}$ F. Yumiceva, ${ }^{155}$ M. R. Adams, ${ }^{156}$ L. Apanasevich, ${ }^{156}$ D. Berry,${ }^{156}$ R. R. Betts ${ }^{156}$ R. Cavanaugh, ${ }^{156}$ X. Chen, ${ }^{156}$ S. Dittmer, ${ }^{156}$ O. Evdokimov, ${ }^{156}$ C. E. Gerber, ${ }^{156}$ D. A. Hangal ${ }^{156}$ D. J. Hofman, ${ }^{156}$ K. Jung, ${ }^{156}$ C. Mills, ${ }^{156}$ M. B. Tonjes, ${ }^{156}$ N. Varelas, ${ }^{156}$ H. Wang, ${ }^{156}$ X. Wang, ${ }^{156}$ Z. Wu, ${ }^{156}$ J. Zhang, ${ }^{156}$ M. Alhusseini, ${ }^{157}$ B. Bilki, ${ }^{157, d d d}$ W. Clarida, ${ }^{157}$ K. Dilsiz, ${ }^{157, \text { uu }}$ S. Durgut, ${ }^{157}$ R. P. Gandrajula, ${ }^{157}$ M. Haytmyradov, ${ }^{157}$ V. Khristenko, ${ }^{157}$ O. K. Köseyan, ${ }^{157}$ J.-P. Merlo, ${ }^{157}$ A. Mestvirishvili, ${ }^{157}$ A. Moeller ${ }^{157}$ J. Nachtman, ${ }^{157}$ H. Ogul, ${ }^{157, v v v}$ Y. Onel, ${ }^{157}$ F. Ozok, ${ }^{157, w w w}$ A. Penzo, ${ }^{157}$ C. Snyder, ${ }^{157}$ E. Tiras, ${ }^{157}$ J. Wetzel, ${ }^{157}$ B. Blumenfeld, ${ }^{158}$ A. Cocoros, ${ }^{158}$ N. Eminizer, ${ }^{158}$ D. Fehling, ${ }^{158}$ L. Feng, ${ }^{158}$ A. V. Gritsan, ${ }^{158}$ W. T. Hung, ${ }^{158}$ P. Maksimovic, ${ }^{158}$ J. Roskes, ${ }^{158}$ U. Sarica, ${ }^{158}$ M. Swartz, ${ }^{158}$ M. Xiao, ${ }^{158}$ A. Al-bataineh, ${ }^{159}$ P. Baringer, ${ }^{159}$ A. Bean, ${ }^{159}$ S. Boren, ${ }^{159}$ J. Bowen, ${ }^{159}$ A. Bylinkin, ${ }^{159}$ J. Castle, ${ }^{159}$ S. Khalil,${ }^{159}$ A. Kropivnitskaya,${ }^{159}$ D. Majumder, ${ }^{159}$ W. Mcbrayer, ${ }^{159}$ M. Murray, ${ }^{159}$ C. Rogan, ${ }^{159}$ S. Sanders,${ }^{159}$ E. Schmitz, ${ }^{159}$ J. D. Tapia Takaki, ${ }^{159}$ Q. Wang, ${ }^{159}$ S. Duric, ${ }^{160}$ A. Ivanov, ${ }^{160}$ K. Kaadze, ${ }^{160}$ D. Kim, ${ }^{160}$ Y. Maravin, ${ }^{160}$ D. R. Mendis, ${ }^{160}$ T. Mitchell, ${ }^{160}$ A. Modak, ${ }^{160}$ A. Mohammadi, ${ }^{160}$ F. Rebassoo, ${ }^{161}$ D. Wright, ${ }^{161}$ A. Baden, ${ }^{162}$ O. Baron, ${ }^{162}$ A. Belloni, ${ }^{162}$ S. C. Eno, ${ }^{162}$ Y. Feng, ${ }^{162}$ C. Ferraioli, ${ }^{162}$ N. J. Hadley, ${ }^{162}$ S. Jabeen, ${ }^{162}$ G. Y. Jeng, ${ }^{162}$ R. G. Kellogg, ${ }^{162}$ J. Kunkle, ${ }^{162}$ A. C. Mignerey, ${ }^{162}$ S. Nabili, ${ }^{162}$ F. Ricci-Tam, ${ }^{162}$ M. Seidel, ${ }^{162}$ Y. H. Shin, ${ }^{162}$ A. Skuja, ${ }^{162}$ S. C. Tonwar, ${ }^{162}$ K. Wong, ${ }^{162}$ D. Abercrombie, ${ }^{163}$ B. Allen, ${ }^{163}$ V. Azzolini, ${ }^{163}$ A. Baty, ${ }^{163}$ R. Bi, ${ }^{163}$ S. Brandt, ${ }^{163}$ W. Busza, ${ }^{163}$ I. A. Cali, ${ }^{163}$ M. D’ Alfonso, ${ }^{163}$ G. Gomez Ceballos, ${ }^{163}$ M. Goncharov, ${ }^{163}$ P. Harris, ${ }^{163}$ D. Hsu, ${ }^{163}$ M. Hu,${ }^{163}$ M. Klute, ${ }^{163}$ D. Kovalskyi, ${ }^{163}$ Y.-J. Lee, ${ }^{163}$ P. D. Luckey, ${ }^{163}$ B. Maier, ${ }^{163}$ A. C. Marini, ${ }^{163}$ C. Mcginn, ${ }^{163}$ C. Mironov, ${ }^{163}$ S. Narayanan, ${ }^{163}$ X. Niu, ${ }^{163}$ C. Paus, ${ }^{163}$ D. Rankin, ${ }^{163}$ C. Roland, ${ }^{163}$ G. Roland, ${ }^{163}$ Z. Shi, ${ }^{163}$ G. S. F. Stephans, ${ }^{163}$ K. Sumorok,${ }^{163}$ K. Tatar, ${ }^{163}$ D. Velicanu, ${ }^{163}$ J. Wang, ${ }^{163}$ T. W. Wang, ${ }^{163}$ B. Wyslouch, ${ }^{163}$ A. C. Benvenuti, ${ }^{164, a}$ R. M. Chatterjee, ${ }^{164}$ A. Evans, ${ }^{164}$ P. Hansen, ${ }^{164}$ J. Hiltbrand, ${ }^{164}$ Sh. Jain, ${ }^{164}$ S. Kalafut, ${ }^{164}$ M. Krohn, ${ }^{164}$ Y. Kubota, ${ }^{164}$ Z. Lesko, ${ }^{164}$ J. Mans, ${ }^{164}$ R. Rusack, ${ }^{164}$ M. A. Wadud, ${ }^{164}$ J. G. Acosta, ${ }^{165}$ S. Oliveros, ${ }^{165}$ E. Avdeeva, ${ }^{166}$ K. Bloom, ${ }^{166}$ D. R. Claes, ${ }^{166}$ C. Fangmeier, ${ }^{166}$ L. Finco, ${ }^{166}$ F. Golf,,${ }^{166}$ R. Gonzalez Suarez, ${ }^{166}$ R. Kamalieddin, ${ }^{166}$ I. Kravchenko, ${ }^{166}$ J. E. Siado, ${ }^{166}$ G. R. Snow, ${ }^{166}$ B. Stieger, ${ }^{166}$ A. Godshalk, ${ }^{167}$ C. Harrington, ${ }^{167}$ I. Iashvili, ${ }^{167}$ A. Kharchilava, ${ }^{167}$ C. Mclean,${ }^{167}$ D. Nguyen, ${ }^{167}$ A. Parker, ${ }^{167}$ S. Rappoccio, ${ }^{167}$

B. Roozbahani, ${ }^{167}$ G. Alverson, ${ }^{168}$ E. Barberis,${ }^{168}$ C. Freer, ${ }^{168}$ Y. Haddad ${ }^{168}$ A. Hortiangtham, ${ }^{168}$ G. Madigan, ${ }^{168}$ D. M. Morse, ${ }^{168}$ T. Orimoto, ${ }^{168}$ A. Tishelman-Charny, ${ }^{168}$ T. Wamorkar, ${ }^{168}$ B. Wang, ${ }^{168}$ A. Wisecarver, ${ }^{168}$ D. Wood, ${ }^{168}$ S. Bhattacharya, ${ }^{169}$ J. Bueghly, ${ }^{169}$ T. Gunter, ${ }^{169}$ K. A. Hahn, ${ }^{169}$ N. Odell, ${ }^{169}$ M. H. Schmitt,,${ }^{169}$ K. Sung, ${ }^{169}$ M. Trovato, ${ }^{169}$ M. Velasco, ${ }^{169}$ R. Bucci, ${ }^{170}$ N. Dev, ${ }^{170}$ R. Goldouzian, ${ }^{170}$ M. Hildreth, ${ }^{170}$ K. Hurtado Anampa,${ }^{170}$ C. Jessop,${ }^{170}$

D. J. Karmgard, ${ }^{170}$ K. Lannon, ${ }^{170}$ W. Li, ${ }^{170}$ N. Loukas, ${ }^{170}$ N. Marinelli, ${ }^{170}$ F. Meng, ${ }^{170}$ C. Mueller, ${ }^{170}$ Y. Musienko, ${ }^{170,00}$ M. Planer, ${ }^{170}$ R. Ruchti, ${ }^{170}$ P. Siddireddy, ${ }^{170}$ G. Smith,${ }^{170}$ S. Taroni, ${ }^{170}$ M. Wayne, ${ }^{170}$ A. Wightman, ${ }^{170}$ M. Wolf, ${ }^{170}$ A. Woodard,${ }^{170}$ J. Alimena, ${ }^{171}$ L. Antonelli, ${ }^{171}$ B. Bylsma, ${ }^{171}$ L. S. Durkin, ${ }^{171}$ S. Flowers, ${ }^{171}$ B. Francis, ${ }^{171}$ C. Hill, ${ }^{171}$ W. Ji, ${ }^{171}$ A. Lefeld, ${ }^{171}$ T. Y. Ling, ${ }^{171}$ W. Luo, ${ }^{171}$ B. L. Winer, ${ }^{171}$ S. Cooperstein, ${ }^{172}$ G. Dezoort,${ }^{172}$ P. Elmer, ${ }^{172}$ J. Hardenbrook, ${ }^{172}$ N. Haubrich, ${ }^{172}$ S. Higginbotham, ${ }^{172}$ A. Kalogeropoulos, ${ }^{172}$ S. Kwan, ${ }^{172}$ D. Lange, ${ }^{172}$ M. T. Lucchini, ${ }^{172}$ J. Luo, ${ }^{172}$ D. Marlow, ${ }^{172}$ K. Mei, ${ }^{172}$ I. Ojalvo, ${ }^{172}$ J. Olsen, ${ }^{172}$ C. Palmer, ${ }^{172}$ P. Piroué,${ }^{172}$ J. Salfeld-Nebgen, ${ }^{172}$ D. Stickland, ${ }^{172}$ C. Tully, ${ }^{172}$ Z. Wang, ${ }^{172}$ S. Malik, ${ }^{173}$ S. Norberg, ${ }^{173}$ A. Barker,${ }^{174}$ V. E. Barnes, ${ }^{174}$ S. Das, ${ }^{174}$ L. Gutay, ${ }^{174}$ M. Jones, ${ }^{174}$ A. W. Jung, ${ }^{174}$ A. Khatiwada, ${ }^{174}$ B. Mahakud, ${ }^{174}$ D. H. Miller,${ }^{174}$ G. Negro, ${ }^{174}$ N. Neumeister, ${ }^{174}$ C. C. Peng, ${ }^{174}$ S. Piperov, ${ }^{174}$ H. Qiu, ${ }^{174}$ J. F. Schulte, ${ }^{174}$ J. Sun, ${ }^{174}$ F. Wang,,${ }^{174}$ R. Xiao,${ }^{174}$ W. Xie, ${ }^{174}$ T. Cheng, ${ }^{175}$ J. Dolen, ${ }^{175}$ N. Parashar, ${ }^{175}$ Z. Chen, ${ }^{176}$ K. M. Ecklund, ${ }^{176}$ S. Freed,${ }^{176}$ F. J. M. Geurts, ${ }^{176}$ M. Kilpatrick, ${ }^{176}$ Arun Kumar, ${ }^{176}$ W. Li, ${ }^{176}$ B. P. Padley, ${ }^{176}$ J. Roberts, ${ }^{176}$ J. Rorie, ${ }^{176}$ W. Shi, ${ }^{176}$ A. G. Stahl Leiton, ${ }^{176}$ Z. Tu ${ }^{176}$ A. Zhang, ${ }^{176}$ A. Bodek, ${ }^{177}$ P. de Barbaro, ${ }^{177}$ R. Demina, ${ }^{177}$ Y. t. Duh, ${ }^{177}$ J. L. Dulemba,${ }^{177}$ C. Fallon, ${ }^{177}$ T. Ferbel, ${ }^{177}$ M. Galanti, ${ }^{177}$ A. Garcia-Bellido, ${ }^{177}$ J. Han, ${ }^{177}$ O. Hindrichs, ${ }^{177}$ A. Khukhunaishvili, ${ }^{177}$ E. Ranken, ${ }^{177}$ P. Tan, ${ }^{177}$ R. Taus, ${ }^{177}$ B. Chiarito, ${ }^{178}$ J. P. Chou, ${ }^{178}$ 
Y. Gershtein, ${ }^{178}$ E. Halkiadakis, ${ }^{178}$ A. Hart, ${ }^{178}$ M. Heindl, ${ }^{178}$ E. Hughes, ${ }^{178}$ S. Kaplan, ${ }^{178}$ S. Kyriacou, ${ }^{178}$ I. Laflotte, ${ }^{178}$ A. Lath, ${ }^{178}$ R. Montalvo, ${ }^{178}$ K. Nash, ${ }^{178}$ M. Osherson, ${ }^{178}$ H. Saka, ${ }^{178}$ S. Salur, ${ }^{178}$ S. Schnetzer, ${ }^{178}$ D. Sheffield, ${ }^{178}$ S. Somalwar, ${ }^{178}$ R. Stone, ${ }^{178}$ S. Thomas, ${ }^{178}$ P. Thomassen, ${ }^{178}$ H. Acharya, ${ }^{179}$ A. G. Delannoy, ${ }^{179}$ J. Heideman, ${ }^{179}$ G. Riley, ${ }^{179}$ S. Spanier, ${ }^{179}$ O. Bouhali, ${ }^{180, x x x}$ A. Celik, ${ }^{180}$ M. Dalchenko, ${ }^{180}$ M. De Mattia, ${ }^{180}$ A. Delgado, ${ }^{180}$ S. Dildick, ${ }^{180}$ R. Eusebi, ${ }^{180}$ J. Gilmore, ${ }^{180}$ T. Huang, ${ }^{180}$ T. Kamon, ${ }^{180, y y y}$ S. Luo, ${ }^{180}$ D. Marley, ${ }^{180}$ R. Mueller, ${ }^{180}$ D. Overton, ${ }^{180}$ L. Perniè,${ }^{180}$ D. Rathjens, ${ }^{180}$ A. Safonov, ${ }^{180}$ N. Akchurin, ${ }^{181}$ J. Damgov, ${ }^{181}$ F. De Guio, ${ }^{181}$ P. R. Dudero, ${ }^{181}$ S. Kunori, ${ }^{181}$ K. Lamichhane, ${ }^{181}$ S. W. Lee ${ }^{181}$ T. Mengke, ${ }^{181}$ S. Muthumuni, ${ }^{181}$ T. Peltola,${ }^{181}$ S. Undleeb,${ }^{181}$ I. Volobouev ${ }^{181}$ Z. Wang, ${ }^{181}$ A. Whitbeck, ${ }^{181}$ S. Greene, ${ }^{182}$ A. Gurrola, ${ }^{182}$ R. Janjam, ${ }^{182}$ W. Johns, ${ }^{182}$ C. Maguire, ${ }^{182}$ A. Melo, ${ }^{182} \mathrm{H}$. Ni, ${ }^{182}$ K. Padeken, ${ }^{182}$ F. Romeo, ${ }^{182}$ P. Sheldon, ${ }^{182}$ S. Tuo, ${ }^{182}$ J. Velkovska, ${ }^{182}$ M. Verweij, ${ }^{182}$ Q. Xu, ${ }^{182}$ M. W. Arenton, ${ }^{183}$ P. Barria, ${ }^{183}$ B. Cox,${ }^{183}$ R. Hirosky, ${ }^{183}$ M. Joyce, ${ }^{183}$ A. Ledovskoy, ${ }^{183}$ H. Li, ${ }^{183}$ C. Neu, ${ }^{183}$ Y. Wang,${ }^{183}$ E. Wolfe, ${ }^{183}$ F. Xia ${ }^{183}$ R. Harr, ${ }^{184}$ P. E. Karchin, ${ }^{184}$ N. Poudyal, ${ }^{184}$ J. Sturdy, ${ }^{184}$ P. Thapa, ${ }^{184}$ S. Zaleski, ${ }^{184}$ J. Buchanan, ${ }^{185}$ C. Caillol, ${ }^{185}$ D. Carlsmith, ${ }^{185}$ S. Dasu, ${ }^{185}$ I. De Bruyn, ${ }^{185}$ L. Dodd, ${ }^{185}$ B. Gomber, ${ }^{185, z z z}$ M. Grothe, ${ }^{185}$ M. Herndon, ${ }^{185}$ A. Herve, ${ }^{185}$ U. Hussain,${ }^{185}$ P. Klabbers, ${ }^{185}$ A. Lanaro, ${ }^{185}$ K. Long, ${ }^{185}$ R. Loveless, ${ }^{185}$ T. Ruggles, ${ }^{185}$ A. Savin, ${ }^{185}$ V. Sharma, ${ }^{185}$ N. Smith, ${ }^{185}$ W. H. Smith, ${ }^{185}$ and N. Woods ${ }^{185}$

(CMS Collaboration)

\footnotetext{
${ }^{1}$ Yerevan Physics Institute, Yerevan, Armenia

${ }^{2}$ Institut für Hochenergiephysik, Wien, Austria

${ }^{3}$ Institute for Nuclear Problems, Minsk, Belarus

${ }^{4}$ Universiteit Antwerpen, Antwerpen, Belgium

${ }^{5}$ Vrije Universiteit Brussel, Brussel, Belgium

${ }^{6}$ Université Libre de Bruxelles, Bruxelles, Belgium ${ }^{7}$ Ghent University, Ghent, Belgium

${ }^{8}$ Université Catholique de Louvain, Louvain-la-Neuve, Belgium

${ }^{9}$ Centro Brasileiro de Pesquisas Fisicas, Rio de Janeiro, Brazil

${ }^{10}$ Universidade do Estado do Rio de Janeiro, Rio de Janeiro, Brazil

${ }^{11 a}$ Universidade Estadual Paulista, São Paulo, Brazil

${ }^{11 \mathrm{~b}}$ Universidade Federal do ABC, São Paulo, Brazil

${ }^{12}$ Institute for Nuclear Research and Nuclear Energy, Bulgarian Academy of Sciences, Sofia, Bulgaria

${ }^{13}$ University of Sofia, Sofia, Bulgaria

${ }^{14}$ Beihang University, Beijing, China

${ }^{15}$ Institute of High Energy Physics, Beijing, China

${ }^{16}$ State Key Laboratory of Nuclear Physics and Technology, Peking University, Beijing, China

${ }^{17}$ Tsinghua University, Beijing, China

${ }^{18}$ Universidad de Los Andes, Bogota, Colombia

${ }^{19}$ Universidad de Antioquia, Medellin, Colombia

${ }^{20}$ University of Split, Faculty of Electrical Engineering, Mechanical Engineering and Naval Architecture, Split, Croatia

${ }^{21}$ University of Split, Faculty of Science, Split, Croatia

${ }^{22}$ Institute Rudjer Boskovic, Zagreb, Croatia

${ }^{23}$ University of Cyprus, Nicosia, Cyprus

${ }^{24}$ Charles University, Prague, Czech Republic

${ }^{25}$ Escuela Politecnica Nacional, Quito, Ecuador

${ }^{26}$ Universidad San Francisco de Quito, Quito, Ecuador

${ }^{27}$ Academy of Scientific Research and Technology of the Arab Republic of Egypt,

Egyptian Network of High Energy Physics, Cairo, Egypt

${ }^{28}$ National Institute of Chemical Physics and Biophysics, Tallinn, Estonia

${ }^{29}$ Department of Physics, University of Helsinki, Helsinki, Finland

${ }^{30}$ Helsinki Institute of Physics, Helsinki, Finland

${ }^{31}$ Lappeenranta University of Technology, Lappeenranta, Finland

${ }^{32}$ IRFU, CEA, Université Paris-Saclay, Gif-sur-Yvette, France

${ }^{33}$ Laboratoire Leprince-Ringuet, Ecole polytechnique, CNRS/IN2P3, Université Paris-Saclay, Palaiseau, France

${ }^{34}$ Université de Strasbourg, CNRS, IPHC UMR 7178, Strasbourg, France

${ }^{35}$ Centre de Calcul de l'Institut National de Physique Nucleaire et de Physique des Particules, CNRS/IN2P3, Villeurbanne, France

${ }^{36}$ Université de Lyon, Université Claude Bernard Lyon 1, CNRS-IN2P3, Institut de Physique Nucléaire de Lyon, Villeurbanne, France

${ }^{37}$ Georgian Technical University, Tbilisi, Georgia
} 


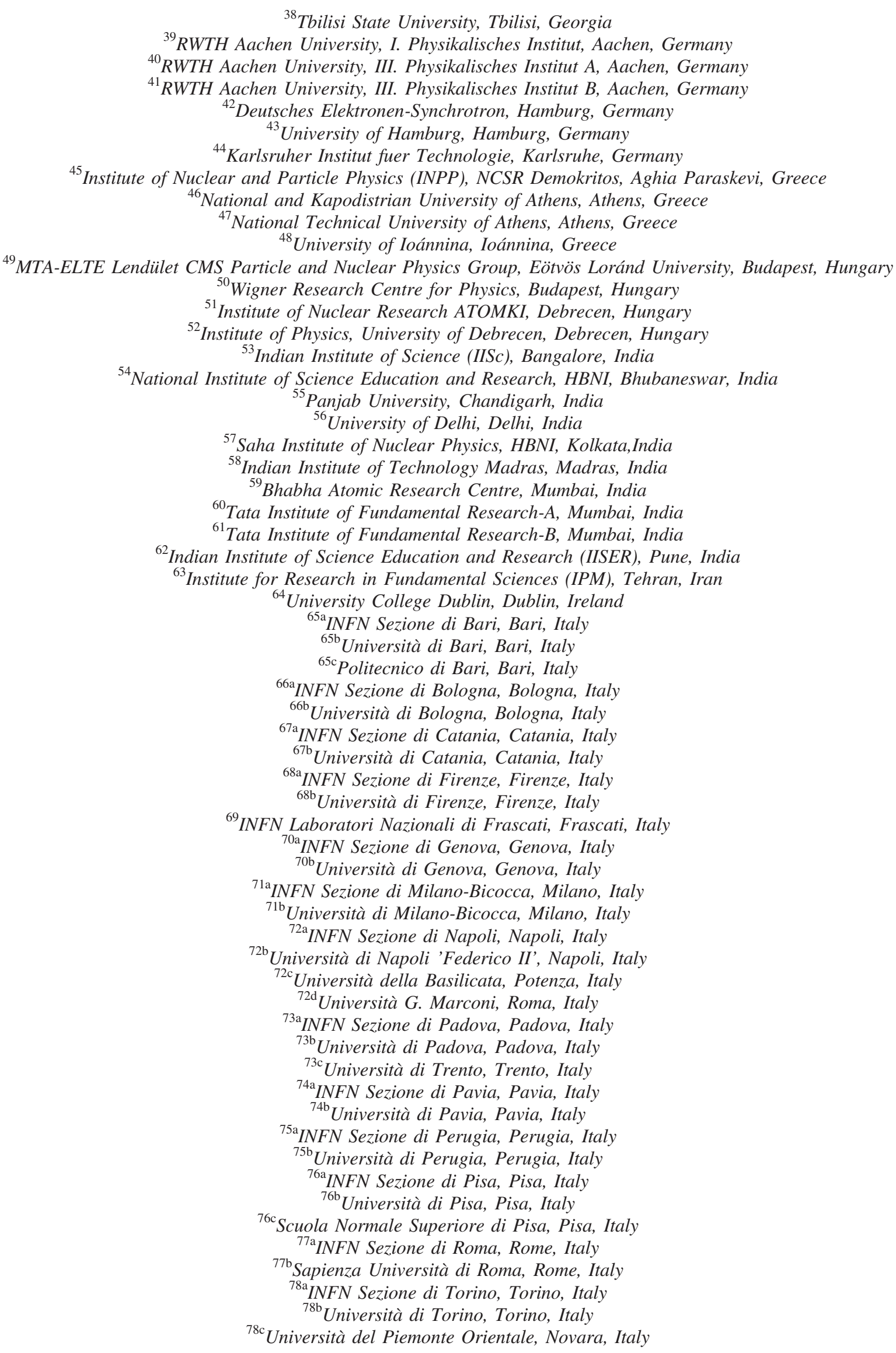




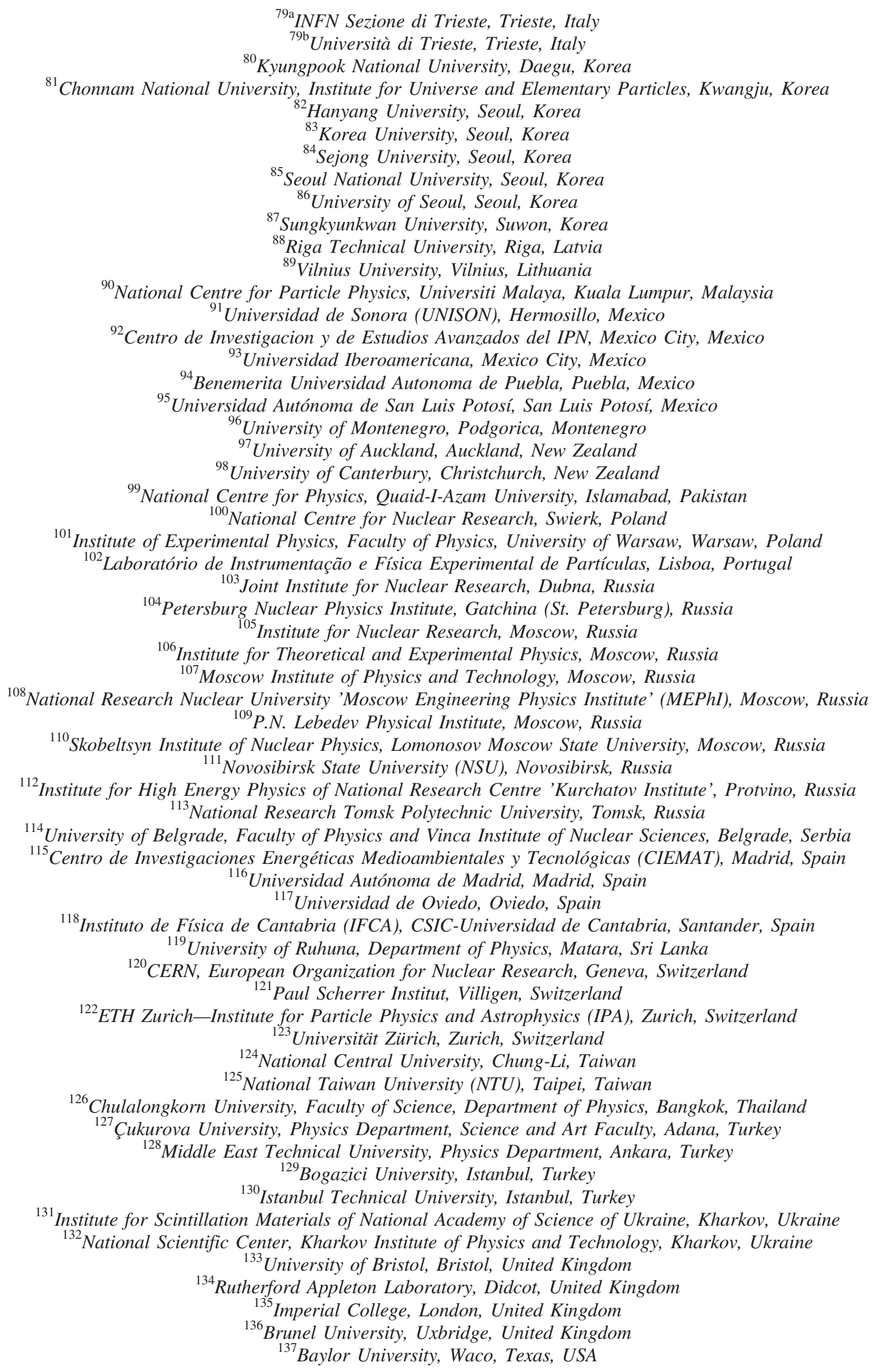


${ }^{138}$ Catholic University of America, Washington, DC, USA

${ }^{139}$ The University of Alabama, Tuscaloosa, Alabama, USA

${ }^{140}$ Boston University, Boston, Massachusetts, USA

${ }^{141}$ Brown University, Providence, Rhode Island, USA

${ }^{142}$ University of California, Davis, Davis, California, USA

${ }^{143}$ University of California, Los Angeles, California, USA

${ }^{144}$ University of California, Riverside, Riverside, California, USA

${ }^{145}$ University of California, San Diego, La Jolla, California, USA

${ }^{146}$ University of California, Santa Barbara-Department of Physics, Santa Barbara, California, USA

${ }^{147}$ California Institute of Technology, Pasadena, California, USA

${ }^{148}$ Carnegie Mellon University, Pittsburgh, Pennsylvania, USA

${ }^{149}$ University of Colorado Boulder, Boulder, Colorado, USA

${ }^{150}$ Cornell University, Ithaca, New York, USA

${ }^{151}$ Fermi National Accelerator Laboratory, Batavia, Illinois, USA

${ }^{152}$ University of Florida, Gainesville, Florida, USA

${ }^{153}$ Florida International University, Miami, Florida, USA

${ }^{154}$ Florida State University, Tallahassee, Florida, USA

${ }^{155}$ Florida Institute of Technology, Melbourne, Florida, USA

${ }^{156}$ University of Illinois at Chicago (UIC), Chicago, Illinois, USA

${ }^{157}$ The University of Iowa, Iowa City, Iowa, USA

${ }^{158}$ Johns Hopkins University, Baltimore, Maryland, USA

${ }^{159}$ The University of Kansas, Lawrence, Kansas, USA

${ }^{160}$ Kansas State University, Manhattan, Kansas, USA

${ }^{161}$ Lawrence Livermore National Laboratory, Livermore, California, USA

${ }^{162}$ University of Maryland, College Park, Maryland, USA

${ }^{163}$ Massachusetts Institute of Technology, Cambridge, Massachusetts, USA

${ }^{164}$ University of Minnesota, Minneapolis, Minnesota, USA

${ }^{165}$ University of Mississippi, Oxford, Mississippi, USA

${ }^{166}$ University of Nebraska-Lincoln, Lincoln, Nebraska, USA

${ }^{167}$ State University of New York at Buffalo, Buffalo, New York, USA

${ }^{168}$ Northeastern University, Boston, Massachusetts, USA

${ }^{169}$ Northwestern University, Evanston, Illinois, USA

${ }^{170}$ University of Notre Dame, Notre Dame, Indiana, USA

${ }^{171}$ The Ohio State University, Columbus, Ohio, USA

${ }^{172}$ Princeton University, Princeton, New Jersey, USA

${ }^{173}$ University of Puerto Rico, Mayaguez, Puerto Rico

${ }^{174}$ Purdue University, West Lafayette, Indiana, USA

${ }^{175}$ Purdue University Northwest, Hammond, Indiana, USA

${ }^{176}$ Rice University, Houston, Texas, USA

${ }^{177}$ University of Rochester, Rochester, New York, USA

${ }^{178}$ Rutgers, The State University of New Jersey, Piscataway, New Jersey, USA

${ }^{179}$ University of Tennessee, Knoxville, Tennessee, USA

${ }^{180}$ Texas A\&M University, College Station, Texas, USA

${ }^{181}$ Texas Tech University, Lubbock, Texas, USA

${ }^{182}$ Vanderbilt University, Nashville, Tennessee, USA

${ }^{183}$ University of Virginia, Charlottesville, Virginia, USA

${ }^{184}$ Wayne State University, Detroit, Michigan, USA

${ }^{185}$ University of Wisconsin-Madison, Madison, Wisconsin, USA

${ }^{\mathrm{a}}$ Deceased.

${ }^{\mathrm{b}}$ Also at Vienna University of Technology, Vienna, Austria.

${ }^{\mathrm{c}}$ Also at Skobeltsyn Institute of Nuclear Physics, Lomonosov Moscow State University, Moscow, Russia.

${ }^{\mathrm{d}}$ Also at IRFU, CEA, Université Paris-Saclay, Gif-sur-Yvette, France.

${ }^{\mathrm{e}}$ Also at Universidade Estadual de Campinas, Campinas, Brazil.

${ }^{\mathrm{f}}$ Also at Federal University of Rio Grande do Sul, Porto Alegre, Brazil.

${ }^{g}$ Also at Université Libre de Bruxelles, Bruxelles, Belgium.

${ }^{\mathrm{h}}$ Also at University of Chinese Academy of Sciences, Beijing, China.

${ }^{\mathrm{i}}$ Also at Institute for Theoretical and Experimental Physics, Moscow, Russia.

${ }^{\mathrm{j}}$ Also at Joint Institute for Nuclear Research, Dubna, Russia.

${ }^{\mathrm{k}}$ Also at Cairo University, Cairo, Egypt. 


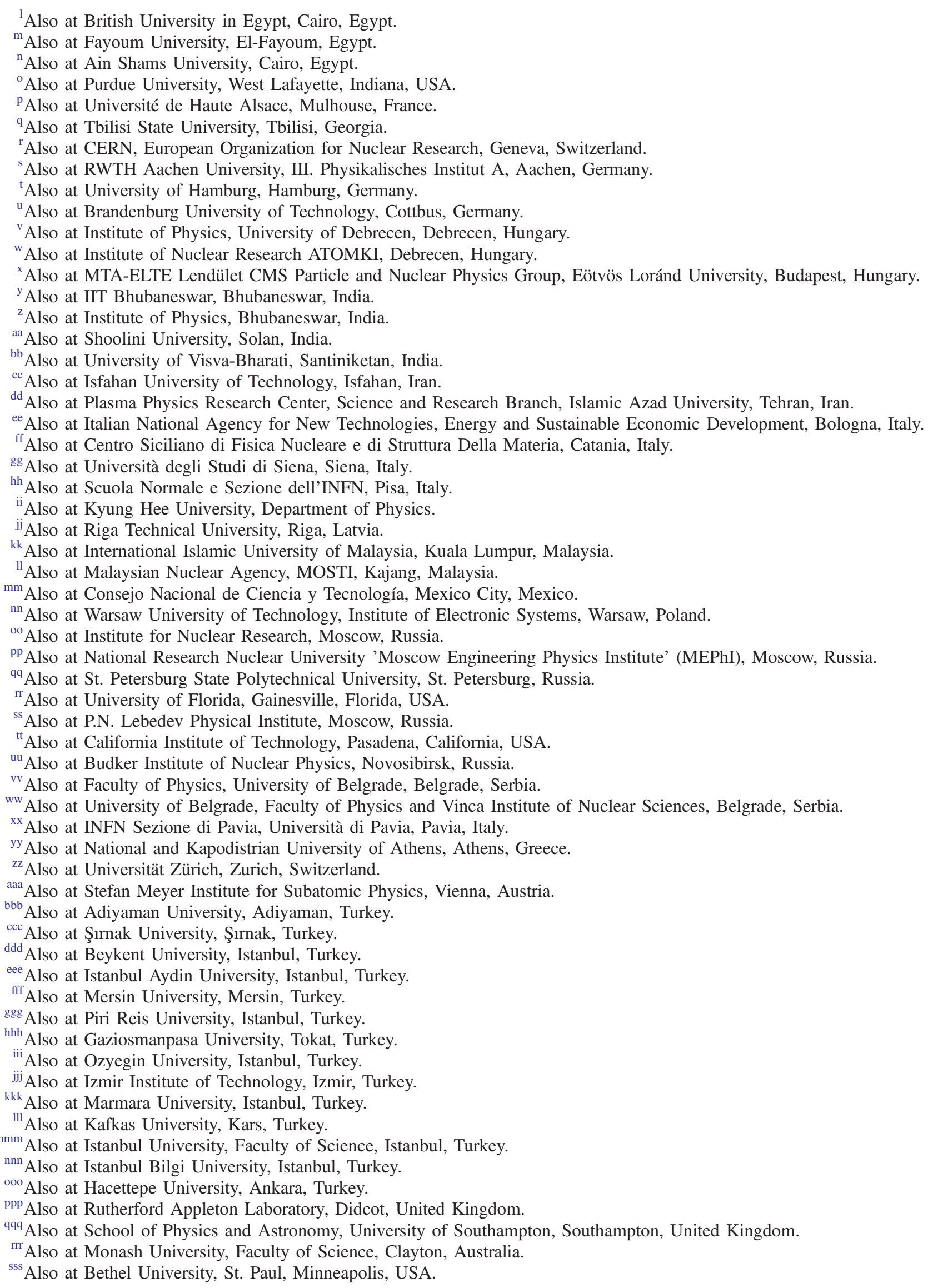




\footnotetext{
${ }^{\text {ttt }}$ Also at Karamanoğlu Mehmetbey University, Karaman, Turkey.

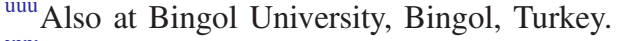

${ }^{\mathrm{vvv}}$ Also at Sinop University, Sinop, Turkey.

${ }^{\text {www }}$ Also at Mimar Sinan University, Istanbul, Istanbul, Turkey.

${ }^{\mathrm{xxx}}$ Also at Texas A\&M University at Qatar, Doha, Qatar.

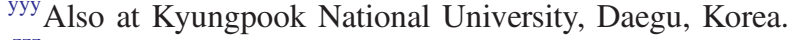

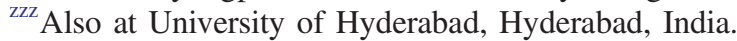

